\title{
Gas structure inside dust cavities of transition disks: Ophiuchus IRS 48 observed by ALMA ${ }^{\star}$
}

\author{
Simon Bruderer ${ }^{1}$, Nienke van der Marel$^{2}$, Ewine F. van Dishoeck ${ }^{2,1}$, and Tim A. van Kempen ${ }^{2}$ \\ 1 Max-Planck-Institut für Extraterrestrische Physik, Giessenbachstrasse 1, 85748 Garching, Germany \\ e-mail: simonbruderer@gmail.com \\ ${ }^{2}$ Leiden Observatory, Leiden University, PO Box 9513, 2300 RA Leiden, The Netherlands
}

Received 16 October 2013 / Accepted 10 December 2013

\section{ABSTRACT}

\begin{abstract}
Context. Transition disks are recognized by the absence of emission of small dust grains inside a radius of up to several 10s of AUs. Owing to the lack of angular resolution and sensitivity, the gas content of these dust holes has not yet been determined, but is of importance for constraining the mechanism leading to the dust holes. It is thought that transition disks are currently undergoing the process of dispersal, setting an end to the giant planet formation process.

Aims. We present new high-resolution observations with the Atacama Large Millimeter/submillimeter Array (ALMA) of gas lines towards the transition disk Oph IRS 48 previously shown to host a large dust trap. The ALMA telescope has detected the $J=6-5$ line of ${ }^{12} \mathrm{CO}$ and $\mathrm{C}^{17} \mathrm{O}$ around $690 \mathrm{GHz}(434 \mu \mathrm{m})$ at a resolution of $\sim 0.25^{\prime \prime}$ corresponding to $\sim 30 \mathrm{AU}$ (FWHM). The observed gas lines are used to set constraints on the gas surface density profile.

Methods. New models of the physical-chemical structure of gas and dust in Oph IRS 48 are developed to reproduce the CO line emission together with the spectral energy distribution and the VLT-VISIR $18.7 \mu \mathrm{m}$ dust continuum images. Integrated intensity cuts and the total spectrum from models having different trial gas surface density profiles are compared to observations. The main parameters varied are the drop in gas surface density inside the dust-free cavity with a radius of $60 \mathrm{AU}$ and inside the gas-depleted innermost $20 \mathrm{AU}$. Using the derived surface density profiles, predictions for other CO isotopologues are made, which can be tested by future ALMA observations of the object.

Results. From the ALMA data we find a total gas mass of the disk of $1.4 \times 10^{-4} M_{\odot}$. This gas mass yields a gas-to-dust ratio of $\sim 10$, but with considerable uncertainty. Inside $60 \mathrm{AU}$, the gas surface density drops by a factor of $\sim 12$ for an assumed surface density slope of $\gamma=1\left(\Sigma \propto r^{-\gamma}\right)$. Inside $20 \mathrm{AU}$, the gas surface density drops by a factor of at least 110 . The drops are measured relative to the extrapolation to small radii of the surface density law at radii $>60 \mathrm{AU}$. The inner radius of the gas disk at $20 \mathrm{AU}$ can be constrained to better than $\pm 5 \mathrm{AU}$.

Conclusions. The derived gas surface density profile points to the clearing of the cavity by one or more massive planets/companions rather than just photoevaporation or grain-growth.
\end{abstract}

Key words. protoplanetary disks - stars: formation - astrochemistry

\section{Introduction}

Protoplanetary disks are the birthplace of young planets. As the disk evolves from a gas-rich $\mathrm{T}$ Tauri disk to a gas-poor debris disk, the disk mass steadily decreases (see Armitage 2011, for a review). The dispersal of the disk sets an end to giant planet formation and thus determines the time-scale of the planet formation process. Detailed understanding of the disk evolution and in particular of the disk dispersal is thus crucial for our understanding of how, where, and when planets form. It is thought that a particular class of protoplanetary disks, called transition disks, is currently in the stage of dispersal. The dust distribution in transition disks has been studied thoroughly through the spectral energy distribution (SED) from ultraviolet (UV) to millimeter wavelengths and in continuum imaging, but still very little is known about the presence and characteristics of the gas in these disks. Here, we use spatially resolved high signal-to-noise gas line observations towards a transition disk to derive the gas structure, and, implicitly determine the origin of the dust holes in this disk.

* Figures $12-15$ are available in electronic form at http://www. aanda.org
Transition disks are commonly identified through their SEDs showing a strong excess over the stellar photosphere at wavelengths $\gtrsim 20 \mu \mathrm{m}$, but little excess at shorter wavelengths. The deficit of near infrared excess arises from the absence of hot small dust close to the star, suggesting the presence of an inner dust cavity (Strom et al. 1989; Calvet et al. 2002; Brown et al. 2007). Several of these dust holes have been imaged directly in (sub)millimeter interferometric imaging (Piétu et al. 2006; Brown et al. 2009; Andrews et al. 2011; Isella et al. 2012, 2013). Recently, candidates for young planets have been found in cavities of the transition disks T Cha by Huélamo et al. (2011), LkCa 15 by Kraus \& Ireland (2012), and HD 100546 by Quanz et al. (2013).

The gas component studied in this work is crucial for distinguishing the different mechanisms proposed for the formation of the dust cavity: grain growth, photo-evaporation, and clearing by a planet or substellar companion. Grain growth does not affect the gas density, while photo-evaporation removes gas and dust simultaneously. Clearing by a planet or substellar companion reduces the amount of gas in the cavity depending on the mass of the companion and other parameters (e.g., viscosity, Zhu et al. 2011; Dodson-Robinson \& Salyk 2011; Pinilla et al. 2012; Mulders et al. 2013; Fung et al. 2013). The 
planet/companion-disk interaction can also induce perturbations in the gas structure leading to local gas pressure maxima. These pressure maxima can prevent large ( $\gtrsim \mathrm{mm}$-sized) dust grains from quickly drifting towards the star before planet formation through dust coagulation and core accretion can take place (e.g., Whipple 1972; Rice et al. 2006; Alexander \& Armitage 2007; Garaud 2007; Kretke \& Lin 2007; Dzyurkevich et al. 2010; Pinilla et al. 2012; Birnstiel et al. 2013; Lyra \& Lin 2013). Thus, the gas structure in transition disks is of direct importance for planet formation. Moreover, the magnitude of any drop in surface density profile is directly related to the mass of the companion.

Gas line observations towards transition disks have been carried out at near infrared and submillimeter wavelengths. The CO rovibrational emission line at $4.7 \mu \mathrm{m}$ tracing the several $100 \mathrm{~K}$ warm gas from the inner regions of the disk has been detected for several transition disks (Goto et al. 2006; Pontoppidan et al. 2008; van der Plas et al. 2009; Salyk et al. 2009; Brown et al. 2012, 2013). In contrast, submillimeter observations of the rotational gas lines trace the bulk of the colder gas and are more suitable for constraining the gas mass and distribution inside the cavity (Bruderer 2013). So far, submillimeter observations have suffered from the low angular resolution and sensitivity, have barely detected the gas in the outer disk, and did not allow the study of properties of the gas inside the cavity (Dutrey et al. 2008; Lyo et al. 2011). With the Atacama Large Millimeter/submillimeter Array $\left(\mathrm{ALMA}^{1}\right)$ the cold gas in the inner regions of transition disks can be imaged for the first time. In this paper, we present a detailed analysis of the gas distribution in the transition disk around the young Herbig star Oph IRS 48, using spatially resolved submillimeter ALMA Band 9 $(690 \mathrm{GHz})$ observations.

Oph IRS $48\left(\alpha_{2000}=16^{\mathrm{h}} 27^{\mathrm{m}} 37^{\mathrm{s}} .18, \delta_{2000}=-24^{\circ} 30^{\prime} 35.3^{\prime \prime}\right)$ is an A0 star located in the $\rho$ Ophiuchi star formation region at a distance of 120 parsec (Loinard et al. 2008; Brown et al. 2012). The star shows weak accretion signatures (Salyk et al. 2013). A ring-like structure peaking at a $\sim 60$ AU radius was found by spatially resolved $18.7 \mu \mathrm{m}$ imaging of the dust continuum (Geers et al. 2007). Centered on the star within this hole, very bright polycyclic aromatic hydrocarbon (PAH) emission is observed towards IRS 48. The VLT-CRIRES spectra of the $4.7 \mu \mathrm{m} \mathrm{CO}$ line reveal a gas ring with a radius of $\sim 25-35 \mathrm{AU}$ (Brown et al. 2012). The submillimeter continuum $(685 \mathrm{GHz}$ or $0.43 \mathrm{~mm})$ of our ALMA observations was presented in van der Marel et al. (2013a). In contrast to the gas and small dust grains, the millimeter dust is concentrated on one side of the disk, with a high azimuthal contrast of $>130$ compared to the other side. This asymmetric dust distribution was interpreted as a dust trap, triggered by the presence of a substellar companion inside $\sim 20$ AU.

In this work we analyze the gas distribution of IRS 48 in detail, using the ALMA observations of ${ }^{12} \mathrm{CO} \mathrm{J}=6-5$ and $\mathrm{C}^{17} \mathrm{O}$ $J=6-5$ taken simultaneously with the continuum observations. Using the combined physical-chemical model by Bruderer et al. (2012) and Bruderer (2013), we derive constraints on the drop in the gas surface density profile at a radius of $60 \mathrm{AU}$, which is the outer radius of the dust cavity, and at $\sim 20 \mathrm{AU}$, where van der Marel et al. (2013a) have identified a gas hole possibly related to a companion situated inside. With the detailed modeling of IRS 48, we also introduce a framework to analyze upcoming ALMA observations of transition disks.

The paper is structured as follows: in Sect. 2 we discuss the observations and data reduction. Section 3 presents spectra,

\footnotetext{
1 http://www . almaobservatory.org
}

integrated intensity maps, and channel maps. In Sect. 4, we present detailed models of IRS 48. We first discuss the dust structure derived from the SED and VISIR images and then compare models with different gas surface density profiles to the ALMA observations. Implications of the derived gas mass and gas surface density structure are given in Sect. 5. The paper ends with a conclusions section.

\section{Observations and data reduction}

Band 9 ALMA Cycle 0 observations of IRS 48 were carried out in the extended configuration on June 6 and July 17, 2012 in three execution blocks of $1.7 \mathrm{~h}$ each (one on June 6th and two on July 17). In these blocks, 18 to 21 antennas were used with baselines up to $390 \mathrm{~m}$. The average precipitable water vapor (PWV) levels were $0.50,0.34$, and $0.17 \mathrm{~mm}$, respectively. The spectral setup consisted of four spectral windows, centered at 674.00, $678.84,691.47$, and $693.88 \mathrm{GHz}$, to target the $\mathrm{C}^{17} \mathrm{O} J=6-5$, $\mathrm{CN} J=6_{11 / 2}-5_{11 / 2},{ }^{12} \mathrm{CO} J=6-5$, and $\mathrm{H}^{13} \mathrm{CO}^{+} J=8-7$ transitions. The $\mathrm{CN}$ and $\mathrm{H}^{13} \mathrm{CO}^{+}$lines have however not been detected and are discussed in van der Marel et al. (2013b) together with a detection of $\mathrm{H}_{2} \mathrm{CO} 9_{18}-8_{17}$. The spectral windows consist of 3840 channels each with a channel separation of 488 $\mathrm{kHz}$ and thus a bandwidth of $1875 \mathrm{MHz}$. The final velocity resolution is $0.21 \mathrm{~km} \mathrm{~s}^{-1}$ using a reference of $690 \mathrm{GHz}$ and the rms noise level is $40 \mathrm{mJy}^{\text {beam }}{ }^{-1}$ channel $^{-1}$. The synthetic beam has a size of $0.32^{\prime \prime} \times 0.21^{\prime \prime}(38 \times 25 \mathrm{AU})$ and a position angle of $96^{\circ}$ (east-of-north).

Reduction and calibration of the data was performed using the Common Astronomy Software Application (CASA) version 3.4 and is further described in the supplementary online material of van der Marel et al. (2013a).

\section{Results}

\subsection{Integrated intensity maps}

Figure 1a shows the continuum subtracted integrated intensity of the ${ }^{12} \mathrm{CO} J=6-5$ observation. The continuum emission at the same wavelength $(430 \mu \mathrm{m})$ is indicated in white contour lines. The strongly lopsided shape of the continuum is interpreted by van der Marel et al. (2013a) as large >mm-size dust being trapped by a local pressure maximum due to a long-lived vortex in the gas, induced by a companion situated at a radius $<20 \mathrm{AU}$. The integrated intensity of $\mathrm{CO}$ is elongated in the east-west direction and shows a drop at the stellar position. The stellar position (Table 1) is determined from the fastest velocity channels where $\mathrm{CO}$ emission has been detected. The elongation is due to the inclination of $i=50^{\circ}$. At the position of the continuum peak, $\mathrm{CO}$ emission is detected, but not particularly strong. This is however not inconsistent with a pressure maximum at this position, because ${ }^{12} \mathrm{CO}$ is likely optically thick and a weak increase in density by less than a factor of 2 is sufficient to trigger dust trapping (Birnstiel et al. 2013; see further discussion in Sect. 5.2).

In Fig. 1b, the continuum subtracted integrated intensity deprojected for inclination, position angle, and distance is shown. After deprojection, the disk is shown face-on with coordinates $(x, y)$ in $\mathrm{AU}$ along the major/minor axis of the observed disk. For the deprojection, the parameters given in Table 1 are used. The inclination is determined by Geers et al. (2007) from $18.7 \mu \mathrm{m}$ dust continuum images to be $i=48 \pm 8^{\circ}$. This inclination is confirmed by Brown et al. (2012) from CO rovibrational lines. We use $i=50^{\circ}$, which was found to agree best with the channel maps of ${ }^{12} \mathrm{CO}$ (Fig. 2). Geers et al. (2007) find a position 

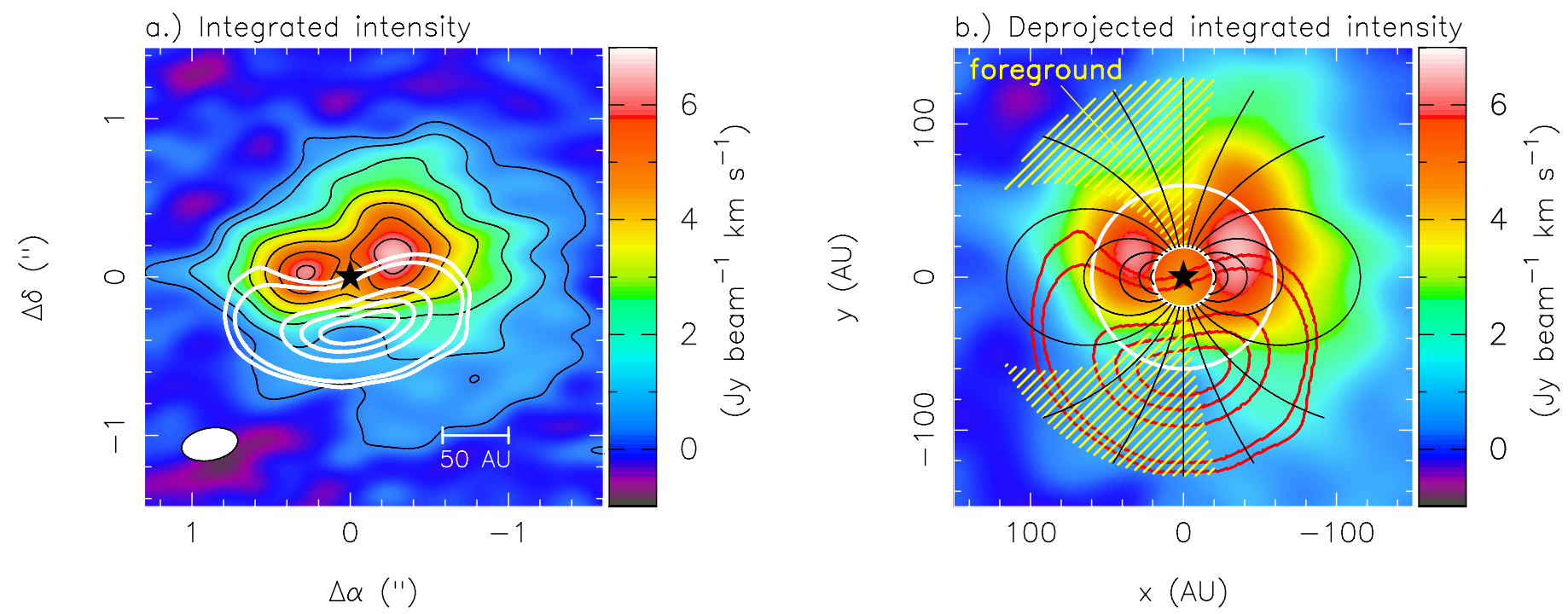

Fig. 1. a) Continuum-subtracted integrated intensity of ${ }^{12} \mathrm{CO} J=6-5$ in color. The black contour lines show $5 \sigma, 10 \sigma, 20 \sigma, \ldots$ detections of the line $\left(1 \sigma=73 \mathrm{mJy}\right.$ beam $\left.^{-1} \mathrm{~km} \mathrm{~s}^{-1}\right)$, white contour lines represent $5 \sigma, 10 \sigma, 100 \sigma, 200 \sigma, 300 \sigma$ detections of the continuum $\left(1 \sigma=0.82 \mathrm{mJy}^{\circ} \mathrm{beam}{ }^{-1}\right)$. The ALMA beam (FWHM) is indicated in the lower left corner. b) Deprojected continuum-subtracted integrated intensity maps of ${ }^{12} \mathrm{CO} J=6-5$ in color and continuum with red contour $(5 \sigma, 10 \sigma, 100 \sigma, 200 \sigma, 300 \sigma$ detections). The $(x, y)$-coordinates are along the major/minor axis of the observed disk. Thick white lines indicate radii of 20 and $60 \mathrm{AU}$ and deprojected isovelocity contours of regions with velocity $\pm 1, \pm 2, \ldots \mathrm{km} \mathrm{s}^{-1}$ relative to $v_{\mathrm{lsr}}$ towards us. The yellow shaded region indicates positions affected by foreground absorption.

Table 1. Basic data of Oph IRS 48.

\begin{tabular}{lcc}
\hline \hline Parameter & Value & Reference \\
\hline Stellar position & $\alpha_{2000}=16^{\mathrm{h}} 27^{\mathrm{m}} 37^{\mathrm{s}} .18$ & $\mathrm{~B} 13$ \\
& $\delta_{2000}=-24^{\circ} 30^{\prime} 35.3^{\prime \prime}$ & \\
Distance & $120 \mathrm{pc}$ & $\mathrm{L} 08$ \\
Inclination & $i=50^{\circ}$ & $\mathrm{G} 07, \mathrm{~B} 13$ \\
Systemic velocity & $v_{\mathrm{lsr}}=4.55 \mathrm{~km} \mathrm{~s}^{-1}$ & $\mathrm{vdM} 13$ \\
Position angle & $\mathrm{PA}=100.3^{\circ}($ east-of-north) & $\mathrm{G} 07, \mathrm{~B} 13$ \\
Stellar type & $\mathrm{A} 0_{-1}^{+4}$ & $\mathrm{~B} 12$ \\
Stellar mass & $M_{*}=2 M_{\odot}$ & $\mathrm{B} 12$ \\
Stellar luminosity & $14.3 L_{\odot}$ & $\mathrm{B} 12$ \\
Accretion rate & $4 \times 10^{-9} M_{\odot} \mathrm{yr}^{-1}$ & $\mathrm{~S} 13$ \\
\hline
\end{tabular}

References. B13 = this work, L08 = Loinard et al. (2008), G07 = Geers et al. (2007), B12 = Brown et al. (2012), S13 = Salyk et al. (2013), vdM13 = van der Marel et al. (2013a).

angle of $98 \pm 3^{\circ}$ (east-of-north) from the $18.7 \mu \mathrm{m}$ dust continuum images. This is consistent with $\mathrm{PA}=100.3^{\circ}$ determined from the position of the highest velocity channels where ${ }^{12} \mathrm{CO}$ is detected (Fig. 2). We overlay deprojected isovelocity contour lines providing lines with the same velocity towards us. The contour lines are derived assuming a geometrically thin disk in Keplerian rotation around a $2 M_{\odot}$ star (Table 1). The deprojected map shows weaker emission in the north-east compared to the north-west. The reason for this is absorption by a foreground cloud. The $\rho$ Ophiuchus region has several foreground layers and clouds with a high enough shielding to be abundant in CO (Loren 1989; Boogert et al. 2002; van Kempen et al. 2009). Such cold foreground $\mathrm{CO}$ can shield the disk emission. The foreground towards IRS $48\left(A_{V}=11.5\right.$, Brown et al. 2012) can provide enough line opacity $(\tau>$ few $)$ in ${ }^{12} \mathrm{CO} J=6-5$ for a cloud with temperatures around $30 \mathrm{~K}$ and densities around $3 \times 10^{4} \mathrm{~cm}^{-3}$. Foreground absorption usually affects only a narrow velocity range, since they are cold and have small intrinsic line widths. Towards IRS 48, velocities $v_{\text {lsr }} \sim 2.0$ to $\sim 4.5 \mathrm{~km} \mathrm{~s}^{-1}$ are affected indicated by the yellow shaded region (van Kempen et al. 2009).

Asymmetries in the east-west direction in the north of the disk ( $y \gtrsim 50 \mathrm{AU}$ in the deprojected map) can thus be explained by foreground absorption. Between $y=-50 \mathrm{AU}$ and $y=50 \mathrm{AU}$, the disk is less extended in the eastern direction, which cannot be explained by the foreground. An additional foreground layer at $v_{\text {lsr }}=1.5 \mathrm{~km} \mathrm{~s}^{-1}$ found towards Oph VLA 1623 by Murillo et al. (2013) would help to explain this asymmetry, but it is unclear if this previously unknown layer also extends to IRS 48. The projected distance of IRS 48 to VLA 1623 is $\sim 19^{\prime}$ or 0.7 pc. At $x \lesssim-20 \mathrm{AU}$, an asymmetry in north-south direction is outside the region affected by foreground by more than the size of the beam of 20-30 AU. For example at $x=-20 \mathrm{AU}$, the emission at $y=-60 \mathrm{AU}$ is about a factor of 3 weaker compared to $y=60 \mathrm{AU}$. At these positions the peak intensities are $1.7 \mathrm{Jy} \mathrm{beam}^{-1}$ and $0.7 \mathrm{Jybeam}^{-1}$, which is stronger than the continuum peak of $0.3 \mathrm{Jy}_{\text {beam }}^{-1}$. Thus, a pure line-tocontinuum effect can also not explain this asymmetry. Since also the peak integrated intensity is slightly shifted to the north with respect to the major axis of the disk ( $y=0 \mathrm{AU})$, a north-south temperature gradient could be the reason for this asymmetry (see further discussion in Sect. 5.2).

We conclude that some, but not all, of the asymmetries seen in the integrated intensity maps can be attributed to foreground absorption. Least affected by the foreground are regions close to the major axis of the disk.

\subsection{Channel maps}

Channel maps of ${ }^{12} \mathrm{CO} 6-5$, binned to $1 \mathrm{~km} \mathrm{~s}^{-1}$, are presented in Fig. 2. Overlaid on the spectrum are isovelocity contours for the velocity bin, derived in the same way as for Fig. 1b (Sect. 3.1). The blue part at $v_{\text {lsr }}<v_{\text {source }}=4.55 \mathrm{~km} \mathrm{~s}^{-1}$ shows in the fastest channels with $\mathrm{CO}$ detected the expected Keplerian pattern, derived using the parameters in Table 1. At slower channels $\left(v_{\mathrm{lsr}}=1.55-2.55 \mathrm{~km} \mathrm{~s}^{-1}\right)$ some emission along the major axis of 


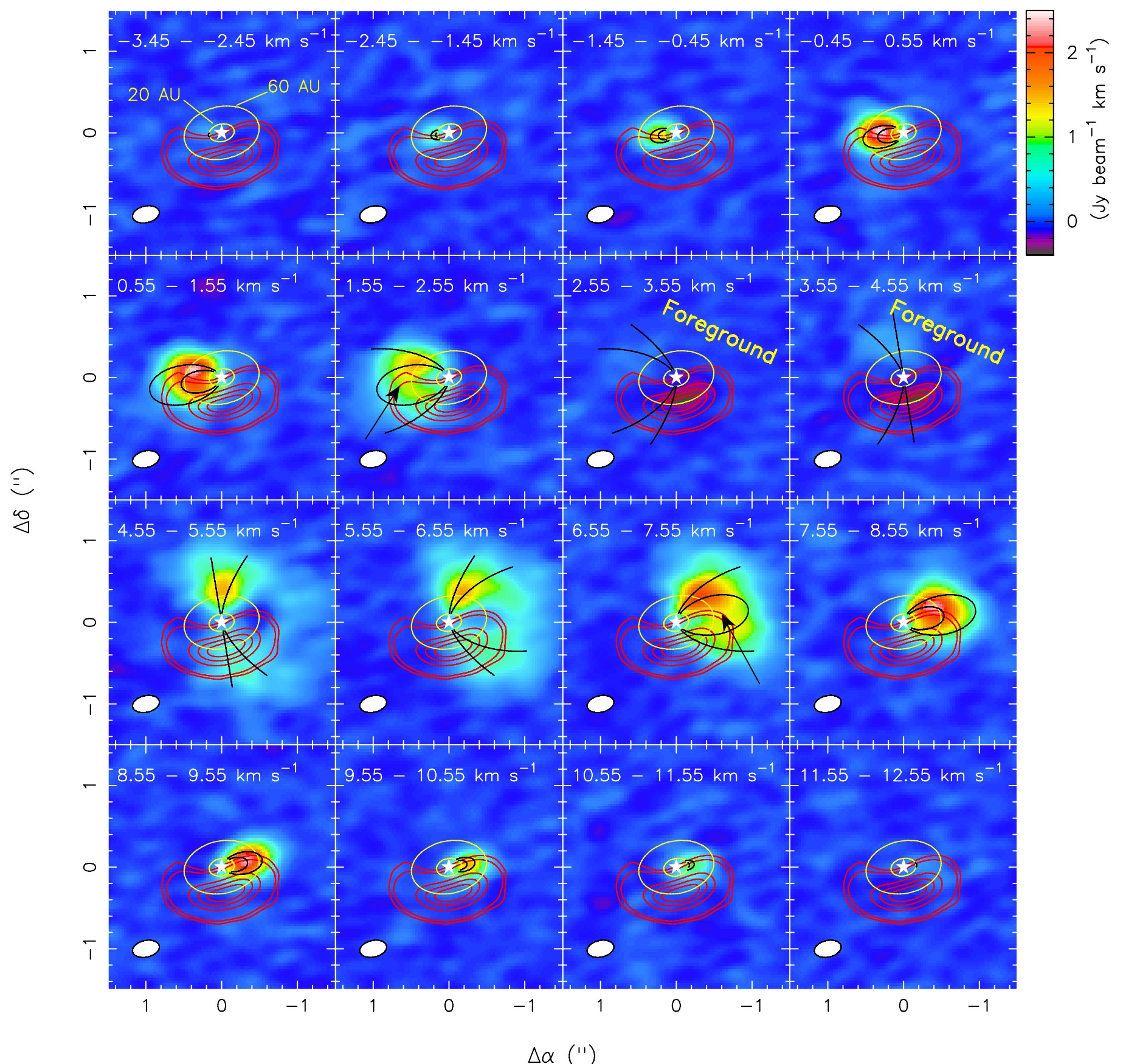

Fig. 2. ${ }^{12} \mathrm{CO} J=6-5$ channel map around $v_{\text {source }}=4.55 \mathrm{~km} \mathrm{~s}^{-1}$. Channels are binned to $1 \mathrm{~km} \mathrm{~s}^{-1}$. Black lines show isovelocity contours of the border velocity of each velocity bin assuming Keplerian rotation with parameters in Table 1. Channels between 2.55 and $4.55 \mathrm{~km} \mathrm{~s}^{-1}$ are affected by foreground absorption. Black arrows indicate regions with clear deviations from the Keplerian pattern (see Sect. 3.2). Ellipses indicate radii of 20 and $60 \mathrm{AU}$. Red contours give the continuum $(5 \sigma, 10 \sigma, 100 \sigma, 200 \sigma, 300 \sigma$ detections).

the disk at distances smaller than expected from the Keplerian pattern is found (indicated by black arrows in Fig. 2). The reason for this slow gas is not clear, but could be related to gas streaming inwards. In our data, this component is only clearly seen at distances $>60 \mathrm{AU}$ and it is thus uncertain if it is related to gas streaming towards the star as found by Casassus et al. (2013) for the case of HD 142527. Between $v_{\mathrm{lsr}}=2.55$ and $4.55 \mathrm{~km} \mathrm{~s}^{-1}$, the emission is completely absorbed by foreground. The red-shifted channels with respect to $v_{\text {source }}$ show a Keplerian pattern for channels between $v_{\mathrm{lsr}}=4.55-6.55 \mathrm{~km} \mathrm{~s}^{-1}$ and the fastest channels $\left(v_{\mathrm{lsr}}>8.55 \mathrm{~km} \mathrm{~s}^{-1}\right)$. In between, some emission along the major axis at smaller distances than expected from the Keplerian pattern is found, as for the blue shifted side.
We conclude that the disk for higher velocities and thus for regions closer to the star, follows the expected Keplerian rotation. Deviations from the Keplerian rotation are found at velocities slower than $4 \mathrm{~km} \mathrm{~s}^{-1}$ relative to $v_{\text {source }}$ at regions outside $\sim 60$ AU.

\subsection{Total spectrum and $\mathrm{C}^{17} \mathrm{O}$}

The total spectrum extracted from regions with $>5 \sigma$ detections in ${ }^{12} \mathrm{CO}$ is shown in Fig. 3, together with the spectrum mirrored at $v_{\text {source }}$. Original and mirrored spectrum overlay for velocities faster than $\sim 4 \mathrm{~km} \mathrm{~s}^{-1}$ relative to $v_{\text {source, }}$ indicating a 


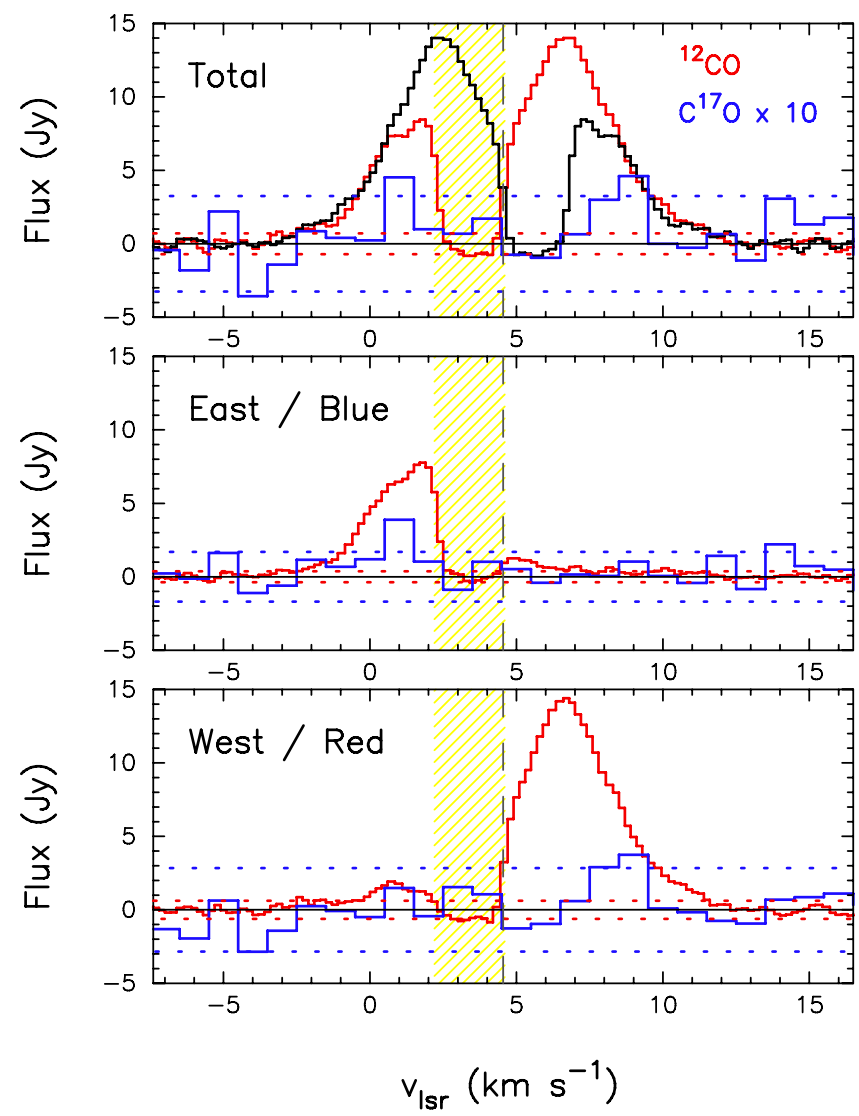

Fig. 3. Continuum-subtracted spectra of ${ }^{12} \mathrm{CO} J=6-5$ (red lines) and ${ }^{17} \mathrm{CO} J=6-5$ (blue lines). The spectra are extracted from regions with $>5 \sigma$ detections in ${ }^{12} \mathrm{CO}$. The yellow shaded region indicates velocities affected by foreground absorption. The vertical dashed line at $4.55 \mathrm{~km} \mathrm{~s}^{-1}$ gives $v_{\text {source}}$. Dotted horizontal lines show the $3 \sigma$ detection level. Top panel: total spectrum. The spectrum of ${ }^{12} \mathrm{CO}$ mirrored on $v_{\text {source }}$ is given in black line. Center panel: spectrum extracted from the east/blue part of the disk. Bottom panel: spectrum extracted from the west/red part of the disk.

symmetric disk close to the protostar. Assuming Keplerian rotation, a velocity shift of $4 \mathrm{~km} \mathrm{~s}^{-1}$ corresponds to radius of $r=\sin (i)^{2} G M_{*} / v^{2} \sim 65 \mathrm{AU}$. CO is detected out to velocity shifts of $\sim 7 \mathrm{~km} \mathrm{~s}^{-1}$, corresponding to a radius of $\sim 20$ AU. Between $v_{\mathrm{lsr}}=0$ and $2 \mathrm{~km} \mathrm{~s}^{-1}$, the blue shifted side is weaker than the red shifted side. This could be due to an additional foreground layer (Sect. 3.1)

The emission of $\mathrm{C}^{17} \mathrm{O}$, observed in parallel with ${ }^{12} \mathrm{CO}$, is too weak to be detected in a single beam. When integrated over a larger region and binned to $1 \mathrm{~km} \mathrm{~s}^{-1}$ velocity-resolution, it has been detected at a $\sim 4 \sigma$ level. Only channels with velocity of 3.5 and $4.5 \mathrm{~km} \mathrm{~s}^{-1}$ relative to $v_{\text {source }}$ are detected. Only the channel at $4.5 \mathrm{~km} \mathrm{~s}^{-1}$ is detected on both the blue and red side. The largest contribution to this channel is from radii of 60 to $110 \mathrm{AU}$, assuming Keplerian rotation. The lower panels in Fig. 3 show spectra extracted from regions where ${ }^{12} \mathrm{CO}$ is detected at a $>5 \sigma$ level in the blue and red part of the spectrum. Indeed, the $\mathrm{C}^{17} \mathrm{O}$ emission is also only detected on the corresponding side of the spectrum, providing some evidence that the $\mathrm{C}^{17} \mathrm{O}$ detection is real.

We conclude from the total spectrum that the disk does not show clear signs of asymmetries inside 60 AU. Observations at higher angular resolution or better sensitivity may however

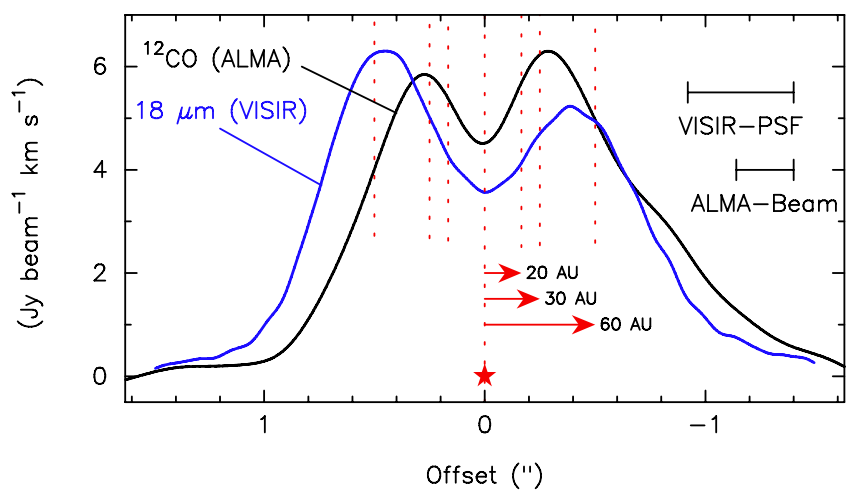

Fig. 4. Integrated intensity of ${ }^{12} \mathrm{CO} J=6-5$ (black line) extracted from a cut through the major axis of the disk ( $y=0 \mathrm{AU}$ in Fig. 1b). The $18.7 \mu \mathrm{m}$ dust emission observed by VLT-VISIR (blue line) has been scaled to the ${ }^{12} \mathrm{CO}$ peak.

reveal asymmetries also in this region. We further conclude that $\mathrm{C}^{17} \mathrm{O}$ is detected at $>60 \mathrm{AU}$, although at a very weak level.

\subsection{A cut through the major axis of the disk}

The previous sections have shown that a cut through the major axis of the disk is least affected by foreground absorption. In Fig. 4, the integrated intensity of ${ }^{12} \mathrm{CO}$ along this cut is presented together with the $18.7 \mu \mathrm{m}$ dust continuum emission observed with VLT-VISIR.

Both the ${ }^{12} \mathrm{CO}$ integrated intensity and $18.7 \mu \mathrm{m}$ dust continuum emission profiles are double-peaked, with the peaks located symmetrically with respect to the star. The strengths of the peaks are slightly asymmetric with the dust emission $\sim 15 \%$ stronger in the east, while the line emission is about $\sim 10 \%$ stronger in the west. The ${ }^{12} \mathrm{CO}$ emission peaks at a distance of $\sim 35 \mathrm{AU}$ to the star, while the dust emission peaks at $\sim 55 \mathrm{AU}$. At the positions where the dust emission peaks, no clear break or change in the ${ }^{12} \mathrm{CO}$ emission can be seen. The depletion in the center of the ${ }^{12} \mathrm{CO}$ emission has about the width of one beam.

\section{Analysis}

In order to constrain changes of the gas surface density profile in particular the drop in the gas surface density inside the dust cavity and the inner gas hole, we use the combined physicalchemical model DALI (Dust And LInes) by Bruderer (2013), based on Bruderer et al. (2012). The models solve for the dust radiative transfer, the chemical abundance, the molecular excitation, and the thermal balance to obtain the gas temperature in a self-consistent way. Such a model is also needed to determine the abundance of $\mathrm{CO}$ inside and outside the dust cavity since it is not a priori obvious that $\mathrm{CO}$ can survive in the dust-free gas exposed to intense UV radiation from the star. The $\mathrm{CO}$ abundance distribution in the disk, together with the gas temperature distribution, determines the intensity and profile of the CO line emission. Spectral image cubes of the line emission are derived from the models and compared to the observations.

The main focus of our modeling are the regions inside the 60 AU radius dust cavity. As discussed in Sect. 3, this region does not show strong signs of asymmetries and we will restrict our analysis to 2D axisymmetric models. Fully 3D models might ultimately be needed to explain the structure of the outer disk, for example, but they are computationally very demanding. Also, a more detailed analysis will require the additional 


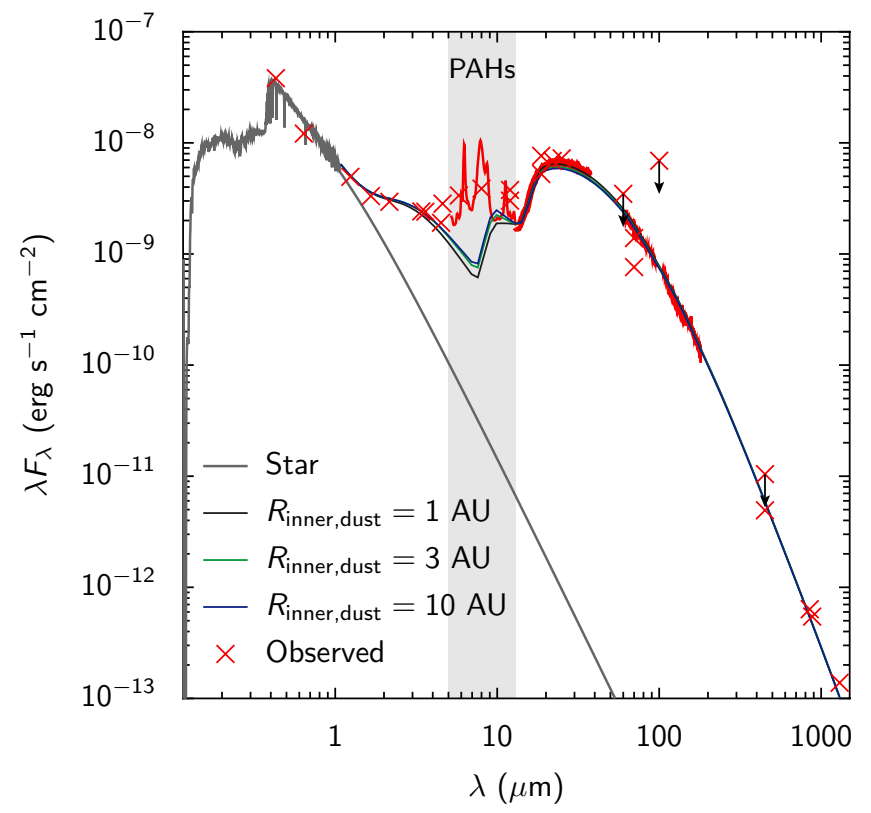

Fig. 5. Observed, dereddened SED of IRS 48 (red crosses/lines) compared to models with different extent (outer radius) of the warm dust inner disk ( $R_{\text {inner,dust }}=1,3$, or $10 \mathrm{AU}$ in black, green, and blue lines). The stellar SED is shown by a grey line. Wavelengths with PAH emission not used in the SED fitting are indicated by a grey shaded region.

constraints from optically thin isotopologue emission (Bruderer 2013) which are not yet available.

In this section, we will first discuss the adopted disk structure for our modeling, and then show the physical and chemical structure derived from the models and a comparison of the line emission to the observations.

\subsection{Disk structure}

The dust structure of IRS 48 is derived from the SED and $18.7 \mu \mathrm{m}$ VLT-VISIR dust continuum image by Geers et al. (2007). The SED is given in Fig. 5 (Table 2). It has been dereddened using a Weingartner \& Draine (2001) extinction curve for $R_{V}=5.5$ assuming $A_{V}=11.5$ (Brown et al. 2012). The stellar SED is derived from the Pickles (1998)-library spectrum for an A0 star with bolometric luminosity $14.3 L_{\odot}$. At wavelengths shorter than $1150 \AA$, this spectrum is extended by FUSE observations towards a star of the same spectral type, scaled for the bolometric luminosity. The dust SED shows excess emission over the stellar emission at near infrared wavelengths $(1-5 \mu \mathrm{m})$, indicative of warm dust close to the star $(<1 \mathrm{AU})$. The spatial location of this warm dust is not known, but is here attributed to the optically thick inner wall of a dusty inner disk. Other SED fits of IRS 48 (Maaskant et al. 2013) have assigned the near infrared excess to a dust halo around the star. A peculiar feature of the SED are the strong PAH features at 5-12 $\mu \mathrm{m}$, discussed by Geers et al. (2007). The mid-infrared excess has been spatially resolved by VLT-VISIR at $18.7 \mu \mathrm{m}$ and comes from a ring located at a radial distance of $\sim 60$ AU to the star. The (sub)millimeter continuum is strongly lopsided, but remains optically thin at $450 \mu \mathrm{m}$ (van der Marel et al. 2013a). The peak emission of the submillimeter continuum is located slightly outside 60 AU (Fig. 1, van der Marel et al. 2013a) and in the following we will assume that both the mid-infrared and (sub)millimeter continuum emission come from an outer disk located at a radius $>60 \mathrm{AU}$.
Table 2. Observed continuum fluxes.

\begin{tabular}{|c|c|c|c|}
\hline Instrument & $\begin{array}{l}\text { Wavelength } \\
\quad(\mu \mathrm{m})\end{array}$ & $\begin{array}{l}\text { Flux } \\
\text { (Jy) }\end{array}$ & Reference \\
\hline \multicolumn{4}{|c|}{ Photometry } \\
\hline NOMAD & 0.43 & $1.60(-5)$ & Zacharias et al. (2005) \\
\hline NOMAD & 0.64 & $3.26(-4)$ & Zacharias et al. (2005) \\
\hline 2MASS & 1.24 & $9.46(-2)$ & Cutri et al. (2003) \\
\hline 2MASS & 1.66 & $3.05(-1)$ & Cutri et al. (2003) \\
\hline 2MASS & 2.16 & $6.18(-1)$ & Cutri et al. (2003) \\
\hline WISE & 3.4 & 1.29 & Wright et al. (2010) \\
\hline IRAC & 3.6 & 1.41 & van Kempen et al. (2009) \\
\hline IRAC & 4.5 & 1.60 & van Kempen et al. (2009) \\
\hline WISE & 4.6 & 2.45 & Wright et al. (2010) \\
\hline IRAC & 5.8 & 4.06 & van Kempen et al. (2009) \\
\hline IRAC & 8.0 & 6.00 & van Kempen et al. (2009) \\
\hline IRAS & 12.0 & 7.81 & Helou \& Walker (1988) \\
\hline WISE & 12.0 & 6.26 & Wright et al. (2010) \\
\hline AKARI & 18.7 & 21.0 & Yamamura et al. (2010) \\
\hline VISIR & 18.7 & 31.8 & Geers et al. (2007) \\
\hline WISE & 22.0 & 36.5 & Wright et al. (2010) \\
\hline IRAS & 25.0 & 46.5 & Helou \& Walker (1988) \\
\hline IRAS & 60.0 & $<65.5$ & Helou \& Walker (1988) \\
\hline MIPS & 70.0 & 17.0 & van Kempen et al. (2009) \\
\hline PACS & 70.0 & 31.0 & Fedele et al. (2013) \\
\hline IRAS & 100.0 & $<225.0$ & Helou \& Walker (1988) \\
\hline ALMA & 450.0 & $9.50(-1)$ & van der Marel et al. (2013a) \\
\hline SCUBA & 450.0 & $<1.57$ & Andrews \& Williams (2007) \\
\hline SCUBA & 850.0 & $1.80(-1)$ & Andrews \& Williams (2007) \\
\hline SMA & 880.0 & $1.60(-1)$ & Andrews \& Williams (2007) \\
\hline SMA & 1300.0 & $6.00(-2)$ & Andrews \& Williams (2007) \\
\hline \multicolumn{4}{|c|}{ Spectroscopy } \\
\hline IRS & $5.9-36.89$ & & McClure et al. (2010) \\
\hline \multirow{4}{*}{ PACS } & \multicolumn{2}{|c|}{$60.0-73.3(\mathrm{~B} 1)$} & Fedele et al. (2013) \\
\hline & \multicolumn{2}{|c|}{$69.8-95.0(\mathrm{~B} 1)$} & Fedele et al. (2013) \\
\hline & \multicolumn{2}{|c|}{$\begin{array}{l}105.0-146.6(\mathrm{R} 1) \\
139.6-180.9(\mathrm{R} 2)\end{array}$} & Fedele et al. (2013) \\
\hline & \multicolumn{2}{|c|}{$139.6-180.9(\mathrm{R} 2)$} & Fedele et al. (2013) \\
\hline
\end{tabular}

Notes. $a(b)$ means $a \times 10^{b}$.

The far-infrared continuum $(60-180 \mu \mathrm{m})$, observed by Herschel (Fedele et al. 2013), is not spatially resolved and it is unknown whether it is ring-like or lopsided.

For our modeling, we adopt the physical structure suggested by Andrews et al. (2011), as implemented by Bruderer (2013). The surface density profile is assumed to be a power-law and the vertical structure follows a Gaussian distribution (vertically isothermal structure). Given that our data do not constrain the vertical structure and disk models assuming hydrostatic equilibrium overestimate the scale-height (Thi et al. 2013), we feel that such a simple approach is warranted. The outer radius of the disk is not the focus of our work, and we here employ a sharp cut-off in the surface density profile rather than an exponential decrease as in Andrews et al. (2011) and Bruderer (2013).

The parameters of the structure are shown in Table 3 and Fig. 6. The surface density profile follows

$\Sigma(r)=\Sigma_{60 \mathrm{AU}} \cdot\left(\frac{r}{60 \mathrm{AU}}\right)^{-\gamma}$

and is defined by the power-law index $\gamma$ and a surface density $\Sigma_{60} \mathrm{AU}$ at $60 \mathrm{AU}$. The surface density profile of the outer disk at $60-160 \mathrm{AU}$ is scaled by different factors within the warm dust inner disk inside $R_{\text {inner,dust }}=1 \mathrm{AU}\left(\delta_{\text {dust }}\right)$, the gas-depleted hole inside $20 \mathrm{AU}$ and the gap between $20-60 \mathrm{AU}\left(\delta_{20} \mathrm{AU}\right.$ and $\left.\delta_{60 \mathrm{AU}}\right)$. In the radial direction, these regions are defined by 
Table 3. Parameters of the representative model.

\begin{tabular}{lcc}
\hline \hline Parameter & & \\
\hline Surface density profile ${ }^{a}$ & $\gamma$ & 1.0 \\
& $\Sigma_{60 \mathrm{AU}, \mathrm{gas}}$ & $3.2 \times 10^{-2} \mathrm{~g} \mathrm{~cm}^{-2}$ \\
& $\Sigma_{60 \mathrm{AU}, \text { dust }}$ & $4.0 \times 10^{-3} \mathrm{~g} \mathrm{~cm}^{-2}$ \\
\hline Radial sizes & Inner warm dust & $0.4-1 \mathrm{AU}$ \\
& Gas-depleted hole & $0.4-20 \mathrm{AU}$ \\
& Dust-free cavity & $1-60 \mathrm{AU}$ \\
& Outer disk & $60-160 \mathrm{AU}$ \\
\hline Scaling of $\Sigma(R)$ & Dust, $0.4-1 \mathrm{AU}$ & $\delta_{\text {dust }}=9 \times 10^{-4}$ \\
& Gas, $0.4-20 \mathrm{AU}$ & $\delta_{20 \mathrm{AU}}<9 \times 10^{-3}$ \\
& Gas, $20-60 \mathrm{AU}$ & $\delta_{60 \mathrm{AU}}=8 \times 10^{-2}$ \\
\hline Vertical structure & $h_{60} \mathrm{AU}$ & $0.14 \mathrm{rad}$ \\
& $\psi$ & 0.22 \\
\hline Dust settling & $f$ & 0.85 \\
& $\chi$ & 0.2 \\
\hline
\end{tabular}

Notes. The parameters are explained in Sect. 4.1 and shown in Fig. 6. (a) See Eq. (1).

the dust sublimation temperature (0.4 AU), the line wings of the ${ }^{12} \mathrm{CO}$ emission (20 AU), and the size of the dust cavity seen in the $18.7 \mu \mathrm{m}$ dust continuum images and the (sub)millimeter continuum (60 AU). The critical parameter to be studied in this work are the drop in the gas surface density profile at $20 \mathrm{AU}$ $\left(\delta_{20 \mathrm{AU}}\right)$ and at $60 \mathrm{AU}\left(\delta_{60 \mathrm{AU}}\right)$.

The vertical distribution is defined by a scale-height angle $h_{60} \mathrm{AU}$ at $60 \mathrm{AU}$, such that the scale-height angle follows $h(r)=h_{60} \mathrm{AU} \cdot(r / 60 \mathrm{AU})^{\psi}$. We use the same dust opacities as Andrews et al. (2011) consisting of a small $(0.005-1 \mu \mathrm{m})$ and large $(0.005 \mu \mathrm{m}-1 \mathrm{~mm})$ population of dust with a (mass) fraction $f$ in large grains and the scale-height of the large grains reduced by a factor $\chi$. We fix $f$ and $\chi$ to the values adopted by Andrews et al. (2011) for other transition disks. A considerably lower value of $f$ is unlikely given the evidence of grain-growth found towards IRS 48 (van der Marel et al. 2013a). Since most of the UV opacity, which controls the gas temperature, is due to the small grain population, the scale height factor $\chi$ of the larger grains does not affect the line emission much.

In order to constrain the dust structure of IRS 48, we have explored the dependence of the model SED on various parameters (e.g., surface density profile, scale-height function). Here, we disregard wavelengths affected by PAH emission $(5-12 \mu \mathrm{m})$ and do not attempt to fit the PAH abundance. For the combined physical-chemical model we adopt a PAH abundance of $\sim 10 \%$ of the ISM abundance (5\% PAH-to-dust mass ratio, Draine \& $\mathrm{Li}$ 2007) derived by comparing the feature-to-continuum ratio provided by Fig. 7 in Geers et al. (2006) to the observed strength of the features.

Exploring the model parameters, we find that reproducing the near infrared excess requires an optically thick dusty inner disk. The amount of excess is determined by the surface of optically thick hot dust at the inner edge of this inner disk. This optically thick layer however shields the wall of the outer disk at $60 \mathrm{AU}$ and decreases the mid-infrared emission. In order to simultaneously fit the near- and mid-infrared emission, the scaleheight of the outer disk needs to be high enough (scale-height angle $h_{60 \mathrm{AU}}=0.14$ radians) to intercept direct stellar irradiation. The disk mass is mostly constrained through the optically thin submillimeter emission. Assuming that the submillimeter emitting dust was originally spread out over the entire disk, the resulting total dust mass is $1.6 \times 10^{-5} M_{\odot}\left(\kappa_{450 \mu \mathrm{m}}=7.1 \mathrm{~cm}^{2} \mathrm{~g}^{-1}\right)$.

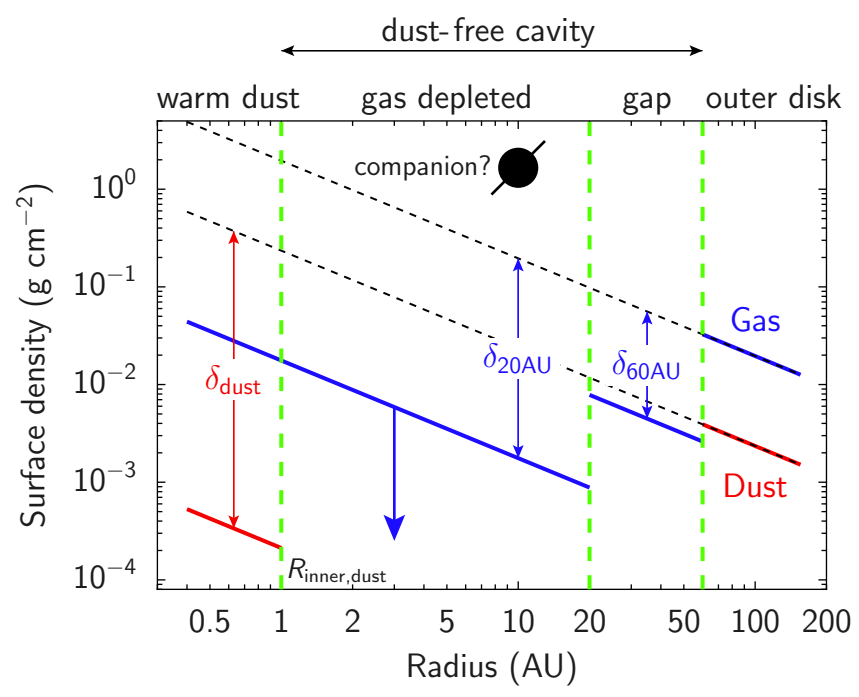

Fig. 6. Surface density profile of the representative model. Gas and dust surface density are given in blue and red lines, respectively. The dashed black lines show the gas/dust surface density if there were no cavities (Eq. (1)). Dashed green lines indicate the outer radii of the inner warm disk, the gas-depleted hole, and the dust-free cavity (Table 3). In the gas-depleted hole, only upper limits on the gas surface density profile can be derived from the observations.

The inner disk mass is with a dust mass of $8 \times 10^{-11} M_{\odot}$ much less massive. The dust mass of the inner disk is not well determined because the near infrared emission traces the optically thick inner wall of the inner disk. The $18.7 \mu \mathrm{m}$ emission of the inner disk is not optically thick and a larger inner disk with a radius of $10 \mathrm{AU}$ and a mass of $1 \times 10^{-9} M_{\odot}$ would overproduce the $18.7 \mu \mathrm{m}$ emission at the stellar position. The submillimeter emission, mapped by ALMA, is not axisymmetric. However, since the dust temperature in the mid-plane remains $>50 \mathrm{~K}$ out to radii of $\sim 120 \mathrm{AU}$ and the submillimeter emission is optically thin, the submillimeter opacities are not important in determining the dust temperature. Thus, our axisymmetric approach does not alter the derived dust mass much.

Figure 7 compares the VLT-VISIR $18.7 \mu$ m observations to model images. The observations show a ring-like feature with a depletion at the stellar position and gradients both in east-west and north-south direction. The gradient in north-south direction is also seen in the models and is the result of the disk's scaleheight in combination with the inclination. The dust emission at $18.7 \mu \mathrm{m}$ in the outer disk is optically thick and traces the surface of the disk. Thus, while both near- and far-side of the northern part of the ring is visible to us, only the near-side is visible to us in the south (Dullemond \& Monnier 2010) resulting in a northsouth asymmetry. Since the inner dusty disk at radii $<10 \mathrm{AU}$ is warm enough to emit at $18.7 \mu \mathrm{m}$, some constraints on the size of this inner dusty disk can be obtained from the image. If the inner disk has an size of 3 or $10 \mathrm{AU}$, the emission at the position of the star is too strong. Hence we choose $R_{\text {inner,dust }}=1$ AU. Varying the radius of the inner disk has little effect on the SED (Fig. 5), since the total emission at this wavelength is dominated by the ring at $\sim 60 \mathrm{AU}$. The reason for the east-west asymmetry is unclear, but could be due to azimuthal changes of the scale-height at the inner disk (Espaillat et al. 2011; Flaherty et al. 2012). 

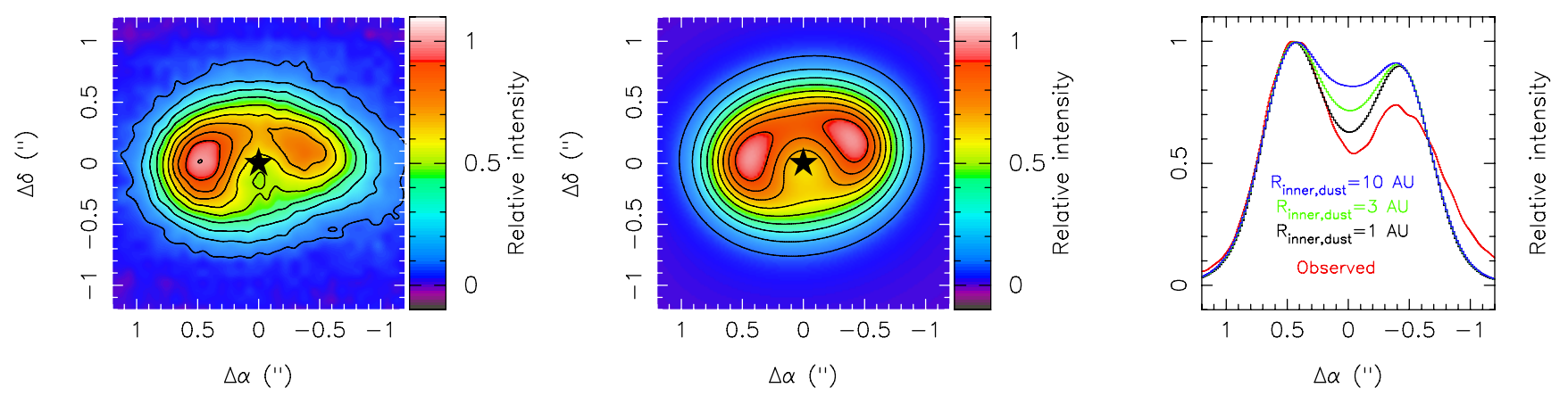

Fig. 7. Left and center panels: observed and modeled VLT-VISIR $18.7 \mu \mathrm{m}$ images. Contour lines show 10, 20, ...\% of the peak emission. Right panel: cut through the observed and modeled images $(\Delta \delta=0)$. Red line: observed intensity. Black, green, and blue lines: modeled intensities for different outer radii of the warm dust inner disk $\left(R_{\text {inner,dust }}=1,3\right.$, or $\left.10 \mathrm{AU}\right)$.
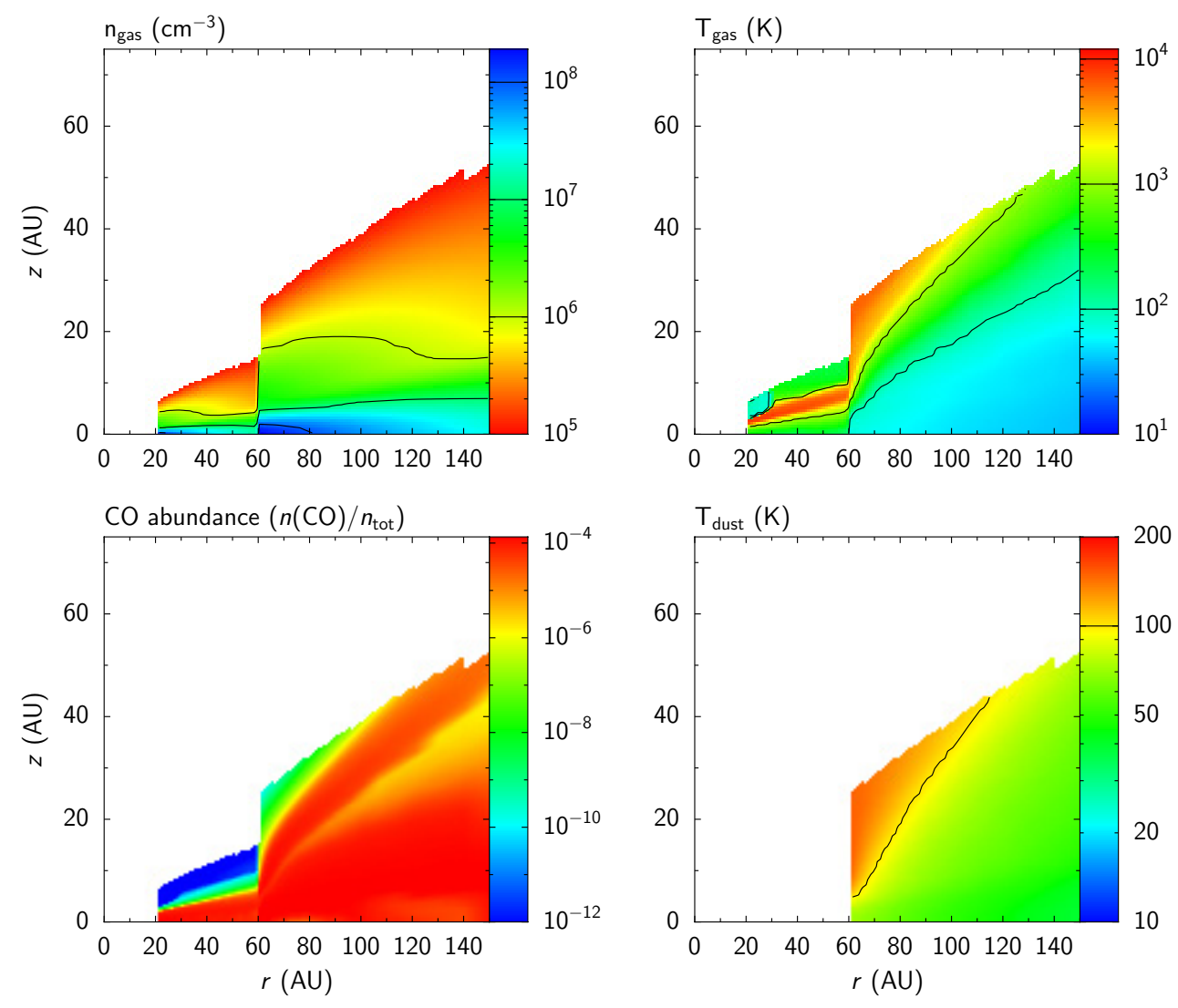

Fig. 8. Density structure, gas and dust temperatures, and $\mathrm{CO}$ fractional abundance of the representative model (Table 3). Only regions with gas density higher than $10^{5} \mathrm{~cm}^{-3}$ are shown. The dust temperature is not defined in the dust-free cavity. The $z$-axis is stretched by a factor of two compared to the $r$-axis.

\subsection{Physical-chemical modeling}

Based on the dust structure derived in the previous section, we run the physical-chemical models for different gas surface density profiles. In the following sections we refer to the representative model as that with the gas surface density following the values given in Fig. 6 and Table 3.

Figure 8 shows the gas density, $\mathrm{CO}$ fractional abundance, gas temperature ( $\left.T_{\text {gas }}\right)$, and dust temperature ( $\left.T_{\text {dust }}\right)$ of the representative model. In this section we focus on the main features of the IRS 48 model, general trends of transition disk models are discussed in Bruderer (2013).

Inside the dust cavity at radii $<60 \mathrm{AU}$, the gas density in the mid-plane reaches $2 \times 10^{8} \mathrm{~cm}^{-3}$. This is far higher than the critical density of CO $6-5$ of $\sim 10^{5} \mathrm{~cm}^{-3}$ and the observed molecular emission of $\mathrm{CO} J=6-5$ is close to local thermal equilibrium (LTE). Temperatures in the dust cavity are several $1000 \mathrm{~K}$ in the upper atmosphere but still above $100 \mathrm{~K}$ in the mid-plane and

thus high enough to excite $\mathrm{CO} 6-5$ with an upper level energy of $115 \mathrm{~K}$. CO is photodissociated in the upper atmosphere by the intense far-ultraviolet radiation of the star. Below a narrow $\mathrm{C}^{+} / \mathrm{CO}$ transition, carbon is fully bound in $\mathrm{CO}$ closer to the midplane.

In the outer disk, the gas and dust temperature close to the mid-plane are well coupled and on the order of $50 \mathrm{~K}$. At higher altitude, the gas temperature decouples from the dust temperature and can reach temperatures of several $1000 \mathrm{~K}$ (Bruderer et al. 2012). This hot layer leads to a "warm finger" of CO, where $\mathrm{CO}$ formation is initiated by the reaction of $\mathrm{C}^{+}$with vibrationally excited $\mathrm{H}_{2}$ (e.g., Jonkheid et al. 2007).

\subsection{Comparison to observations}

To compare model results with observations and to determine the drop in gas surface density at $20 \mathrm{AU}\left(\delta_{20} \mathrm{AU}\right)$ and at $60 \mathrm{AU}$ 
$\left(\delta_{60} \mathrm{AU}\right)$, Fig. 10 shows the ${ }^{12} \mathrm{CO} J=6-5$ integrated intensity cuts along the major axis of the disk for various trial gas distributions. The major parameters that are varied are $\Sigma_{60} \mathrm{AU}, \mathrm{gas}, \delta_{60} \mathrm{AU}$, and $\delta_{20} \mathrm{AU}$. The integrated intensity cut is chosen along the major axis, because this direction is least affected by foreground absorption (Sect. 3.4). In addition to the integrated intensity cut, the total spectrum is presented. The synthetic spectral image cubes from the models are convolved to the ALMA beam. To verify this approach, the result of the representative model convolved to the ALMA beam is compared to the result of simulated ALMA observations (Fig. 14). The ALMA observations have been simulated using the CASA software by converting the model image to $(u, v)$-data according to the observed $(u, v)$-coverage and then reduced in the same way as the observations. Thanks to the good $(u, v)$-coverage of our observations, the two approaches do not differ measurably in the intensity cut and the total spectrum at velocities $\geq 4 \mathrm{~km} \mathrm{~s}^{-1}$ from the source velocity, which is the focus of this work.

The line center opacity of ${ }^{12} \mathrm{CO}$ is on the order of 20 in the center of the dust-free cavity (40 AU) and on the order of 100 in the outer disk at $60 \mathrm{AU}$ for the representative model (Table 3). It is thus clear that the current observations cannot directly constrain the surface density profile (Bruderer 2013). However, due to temperature changes in the vertical structure and the fact that parts of the lines remain optically thin due to the Keplerian rotation, optically thick lines still show some dependence on the gas surface density profile and in particular on changes like drops inside the dust-free cavity. The absolute scaling of the derived surface density profile can be checked with the optically thin emission of $\mathrm{C}^{17} \mathrm{O}$. We do not expect that derived surface density profiles are more accurate than a factor of a few. To test our proposed surface density profile with future observations, we will discuss the derived emission of the optically thin isotopologues in Sect. 4.7.

The disk exhibits asymmetries at radii larger than $60 \mathrm{AU}$ (Sect. 3). Most of the emission from these regions of the disk emerge at low velocities in the spectrum $\left(<4 \mathrm{~km} \mathrm{~s}^{-1}\right.$ relative to $\left.v_{\text {source }}\right)$. We ignore these asymmetries here and focus the model-observation comparison to the inner part of the disk and higher velocities.

\subsubsection{The outer disk ( $\Sigma_{60}$ AU,gas $)$}

Figure 10a shows the ${ }^{12} \mathrm{CO}$ integrated intensity cut and total spectrum of the representative model (Fig. 6 and Table 3) and two models with a factor of 10 increased or decreased gas surface density profile in the outer disk (varied $\Sigma_{60}$ AU,gas). The gas mass is likely dominated by the outer disk, and the outer disk surface density profile thus constrains the total gas mass. The representative model with $\Sigma_{60 \mathrm{AU} \text {,gas }}=3.2 \times 10^{-2} \mathrm{~g} \mathrm{~cm}^{-2}$ (red lines in Fig. 10) yields an integrated intensity along the major axis between the observed east/west integrated intensity. The total spectrum derived from this model also agrees relatively well to the observed spectrum at high velocities $\left(\geq 4 \mathrm{~km} \mathrm{~s}^{-1}\right.$ from the source velocity), corresponding to the inner disk. Slower velocity channels, mostly corresponding to the outer disk, show some deviations. The model emission is too strong at $0.5-2 \mathrm{~km} \mathrm{~s}^{-1}$, possibly due to an additional foreground layer and at $5-8 \mathrm{~km} \mathrm{~s}^{-1}$ due to azimuthal variations of the outer disk (Sect. 3).

Models with a factor of 10 higher column density overproduce both the integrated intensity cut and the total spectrum by more than a factor of two. The model with a factor of 10 lower

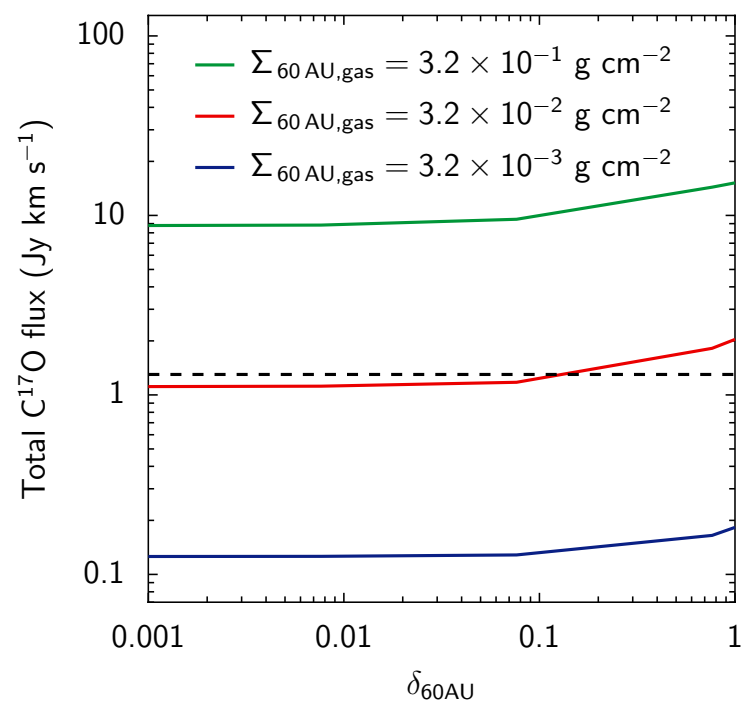

Fig. 9. Total flux of $\mathrm{C}^{17} \mathrm{O}$ for models with $\Sigma_{60} \mathrm{AU}$,gas $=3.2 \times 10^{-1}, 3.2 \times$ $10^{-2}$, and $3.2 \times 10^{-3} \mathrm{~g} \mathrm{~cm}^{-2}$ as a function of the gas surface density at 20-60 AU $\left(\delta_{60} \mathrm{AU}\right)$. The vertical dashed line shows the observed total flux. The red line indicates the representative model.

column density on the other hand, underproduces the integrated intensity at offsets between $0.5^{\prime \prime}$ and $0.8^{\prime \prime}$ (60-100 AU). While the total spectrum of this model better reproduces the wings at around 0.5 and $8.5 \mathrm{~km} \mathrm{~s}^{-1}$, it underproduces the line center.

The $\mathrm{C}^{17} \mathrm{O}$ total flux yields another, independent constraint on the total mass of the disk and thus the surface density profile of the outer disk. Figure 9 compares the modeled $\mathrm{C}^{17} \mathrm{O}$ total flux of the three models discussed before with the observed $1.3 \mathrm{Jy} \mathrm{km} \mathrm{s}^{-1}$. We show the $\mathrm{C}^{17} \mathrm{O}$ flux for three values of $\Sigma_{60 \mathrm{AU} \text {,gas }}$, varying the amount of gas inside $60 \mathrm{AU}\left(\delta_{60 \mathrm{AU}}\right)$. The total gas mass is dominated by the gas mass outside $60 \mathrm{AU}$ for $\delta_{60} \mathrm{AU} \lesssim 0.1$ and rises by only $\sim 50 \%$ for $\delta_{60} \mathrm{AU}=0.1-1$. It is seen that the representative model reproduces the observed flux to within $\sim 20 \%$, while the models with increased/reduced surface density over/underproduce the emission. As $\mathrm{C}^{17} \mathrm{O}$ remains optically thin and thus directly traces the gas mass, its emission scales with the gas mass.

We conclude that the representative model reproduces relatively well the ${ }^{12} \mathrm{CO}$ integrated intensity cut and total spectrum together with the optically thin $\mathrm{C}^{17} \mathrm{O}$ emission. Some deviations in the ${ }^{12} \mathrm{CO}$ total spectrum are due to azimuthal asymmetries of the disk, not included in our model. The gas mass ${ }^{2}$ of the representative model is $1.4 \times 10^{-4} M_{\odot}$. This gas mass is low, only $\sim 15 \%$ of the mass of Jupiter. Combined with the dust mass of $1.6 \times 10^{-5} M_{\odot}$ (Sect. 4.1), this gives a gas-to-dust mass ratio of about 10 . Both gas and dust mass have an uncertainty at a level of a factor of a few.

\subsubsection{Inside the gap ( $\left.\delta_{60} \mathrm{AU}\right)$}

Figure 10b shows the model-observation comparison for models with the surface density profile between 20 and 60 AU varied ( $\delta_{60} \mathrm{AU}$ changed). The representative model is compared to a

2 This gas mass is much lower than that derived by van der Marel et al. (2013a). In that work, we have mistakenly multiplied the number of $\mathrm{H}_{2}$ molecules derived from the $\mathrm{C}^{17} \mathrm{O}$ observations with the mass of $\mathrm{C}^{17} \mathrm{O}$ instead of the $\mathrm{H}_{2}$ mass. 

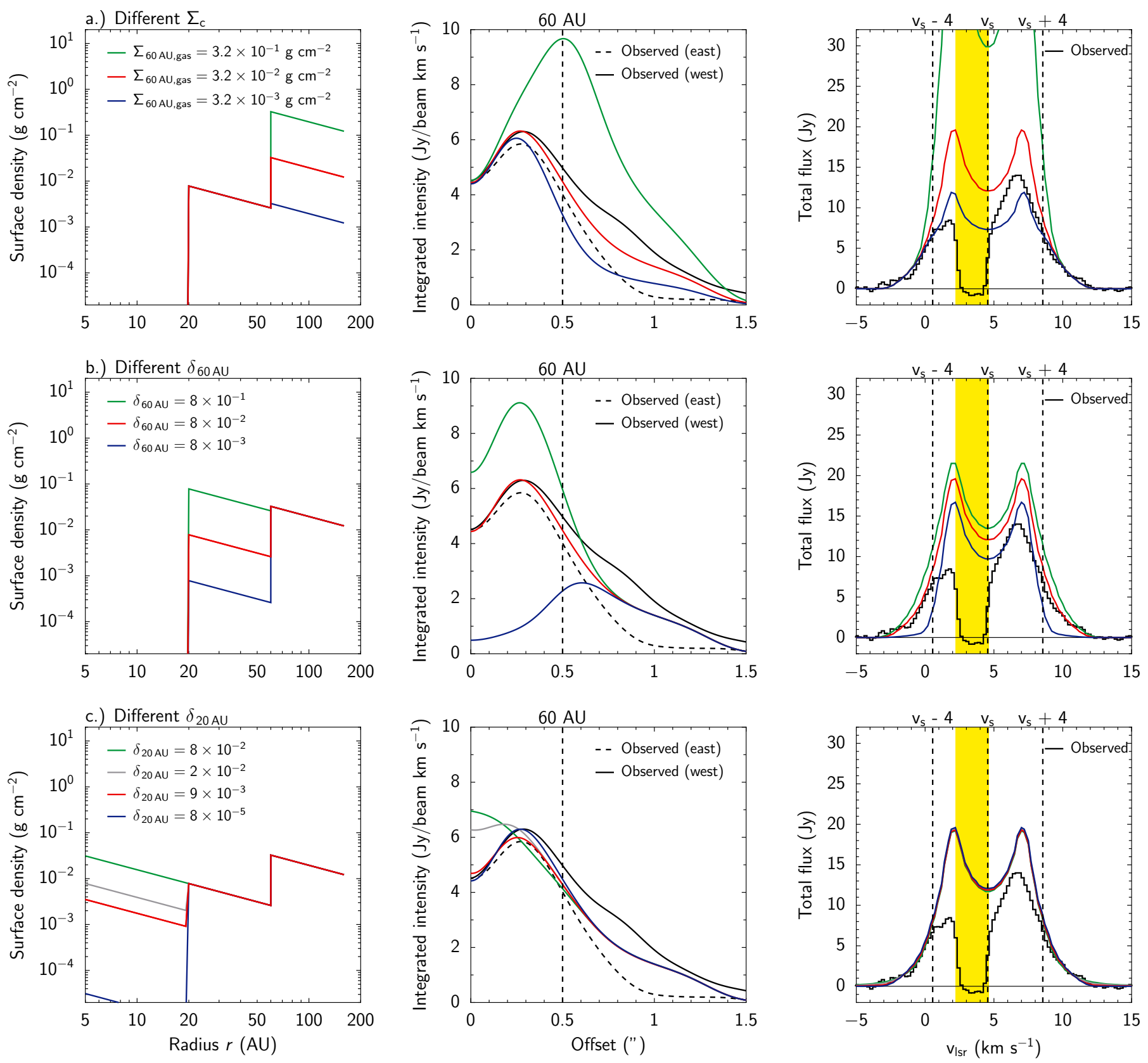

Fig. 10. Comparison of modeled and observed ${ }^{12} \mathrm{CO} J=6-5$ intensity profiles and spectra. Left panels: adopted surface density profiles with variations of the representative model. The red line is the representative model. Center panels: intensity profile extracted along the major axis of the disk. The dashed vertical line indicates the radius of the dust-free cavity. Right panels: total spectrum. The yellow regions indicates regions affected by the foreground. Vertical dashed lines show the source velocity $v_{\mathrm{s}}=4.55 \mathrm{~km} \mathrm{~s}^{-1}$ and velocities $v_{\mathrm{s}} \pm 4 \mathrm{~km} \mathrm{~s}{ }^{-1}$ representing velocity ranges corresponding to the inner disk (radii $\lesssim 60 \mathrm{AU}$ ). a) (Upper row) different surface density profiles in the outer disk (>60 AU). b) (Middle row) different surface density profiles inside the dust gap (20-60 AU). c) (Lower row) Different surface density profiles inside 20 AU.

model with a factor of 10 more/less gas in this region. While the representative model reproduces the integrated intensity cut at offsets $<0.5^{\prime \prime}$ well, the models with a factor of 10 higher/lower surface density at 20-60 AU over/underproduce the integrated intensity. The integrated intensity cut depends on the surface density inside $60 \mathrm{AU}$ out to a radius of $\sim 90 \mathrm{AU}\left(0.75^{\prime \prime}\right)$, due to the width of the beam. The total spectrum of the model with a factor of 10 higher surface density is too broad in the wings and the spectrum with a factor of 10 lower surface density is too narrow.

We conclude that a drop in surface density at 60 AU by about a factor of $\sim 12$ is required to fit the integrated intensity profile and reproduce the line width of the total spectrum.

\subsubsection{The inner $20 \mathrm{AU}\left(\delta_{20} \mathrm{AU}\right)$}

The innermost $20 \mathrm{AU}$ have been suggested by van der Marel et al. (2013a) to be gas-depleted, based on the drop in integrated intensity and the ${ }^{12} \mathrm{CO}$ line wings. They speculated that this drop is related to the presence of an undetected planet or substellar companion. Since the size of this hole is comparable to the beam size (Figs. 1, 2, and 4) and a beam centered on the star will receive some of the much stronger emission from outside $20 \mathrm{AU}$, the current data can only set limits on the gas mass inside $20 \mathrm{AU}$.

Figure 10c shows the representative model with different drops in the gas surface density profile inside $20 \mathrm{AU}$ (varied $\left.\delta_{20} \mathrm{AU}\right)$. In absence of any drop in the gas surface density 
S. Bruderer et al.: Gas structure inside dust cavities of transition disks
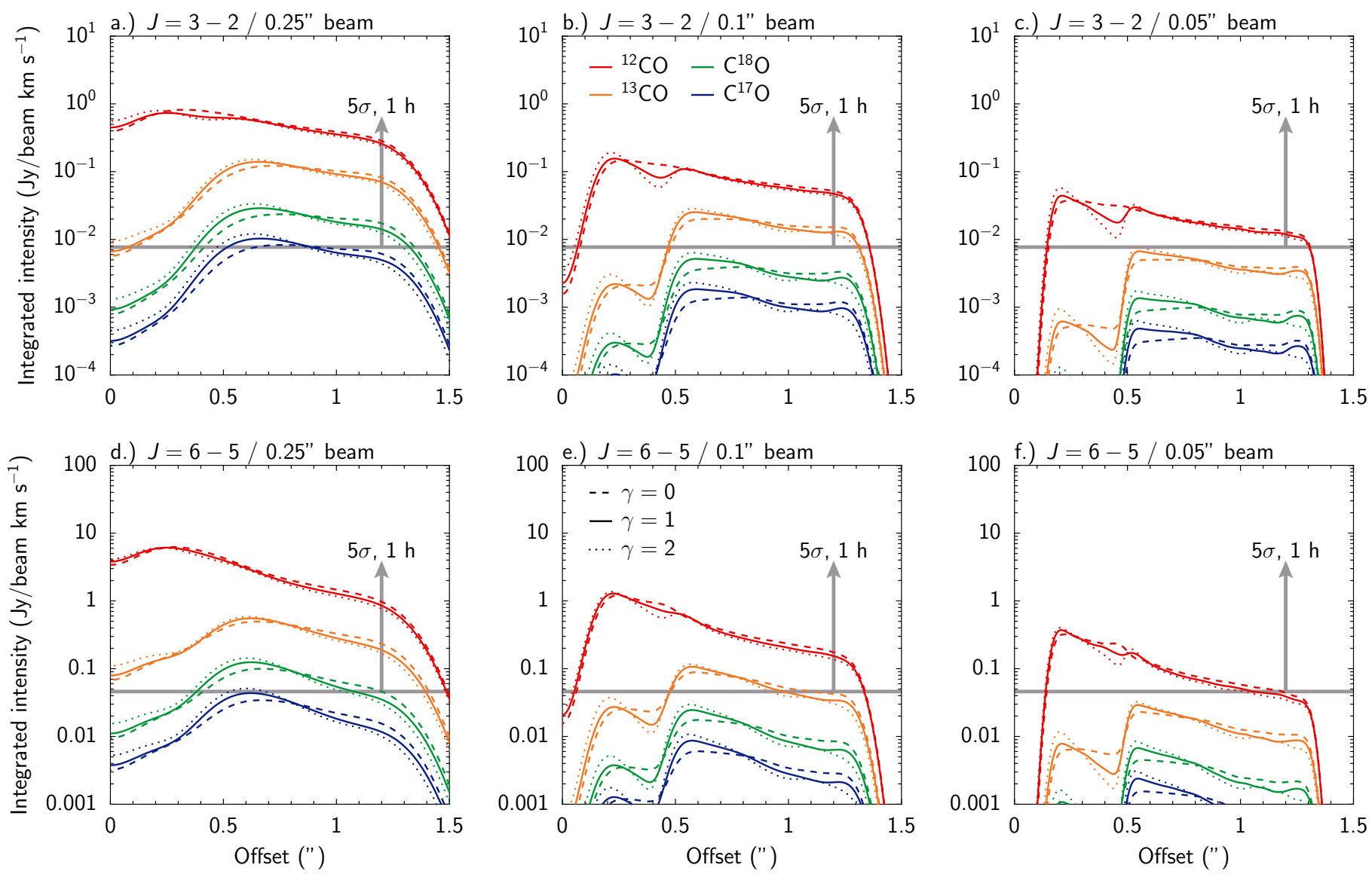

Fig. 11. Beam convolved integrated emission of the $\mathrm{CO}$ isotopologues for the $J=3-2$ and $J=6-5$ transition shown along the cut through the major axis of the disk. The cuts are given for beam sizes of $0.25^{\prime \prime}, 0.1^{\prime \prime}$, and $0.05^{\prime \prime}$. The $5 \sigma$ detection limit within $1 \mathrm{~h}$ of the full ALMA using 50 $12 \mathrm{~m}$ antennas is indicated by the horizontal grey line. Models with a surface density profile slope of $\gamma=0$ (dashed line), $\gamma=1$ (solid line), and $\gamma=2$ (dotted line) are shown.

profile at $20 \mathrm{AU}$, the dip in integrated intensity at the stellar position is not seen. For this amount of gas, the region outside $20 \mathrm{AU}$ is slightly shielded from stellar radiation and the integrated intensity out to an offset of $0.5^{\prime \prime}$ decreases. Only models with a drop in surface density $\delta_{20} \mathrm{AU}<2 \times 10^{-2}$ (factor of $>50$ ) yield a dip in integrated intensity at the stellar position. A model with a drop $\delta_{20 \mathrm{AU}}<9 \times 10^{-3}$ (factor of $>110$ ) reproduces the observed dip at the center position. This model and a model with a drop that is two orders of magnitude larger show very similar integrated intensity cuts and total spectra. Thus, no additional constraints on the gas inside 20 AU can be drawn from the total spectrum.

We conclude that the current data allows us to constrain a drop of a factor of $\gtrsim 110$ in gas surface density profile at 20 AU. This corresponds to a factor of $\gtrsim 10$ drop in surface density profile at $20 \mathrm{AU}$ compared to the surface density at 20 to $60 \mathrm{AU}$. Better constraints will require higher angular resolution observations.

\subsection{The slope $\gamma$ of the surface density profile}

To determine to what extent the assumed slope of the gas and dust surface density profile affects the derived properties, we have also run models with $\gamma=0$ and $\gamma=2$. The SED and $18.7 \mu \mathrm{m}$ images can be equally well fitted for these different values of $\gamma$. Starting from the refitted dust structures, the gas surface density profile has been determined (Fig. 15).
In order to fit the $\mathrm{C}^{17} \mathrm{O}$ emission, the disk mass of the model with $\gamma=0$ and $\gamma=2$ needs to be adjusted to the same value as for the model with $\gamma=1$. For the model with $\gamma=2$ and $\gamma=0$, we constrain $\delta_{60} \mathrm{AU}=3 \times 10^{-2}$ and $\delta_{60} \mathrm{AU}=0.25$, respectively. For $\gamma=0$, the emission at the stellar position is slightly underproduced, while at a distance of $\sim 0.2^{\prime \prime}-0.3^{\prime \prime}$ it is already overproduced. We conclude that the results for the gas mass and the presence of a drop at $60 \mathrm{AU}$ are robust, but that the slope of the surface density profile cannot be well determined by the current data set.

The direct measurement of the surface density slope using $\mathrm{CO}$ and isotopologues is challenging, since different slopes only lead to a factor $\sim 2$ difference in surface density over the range of radii. In Fig. 11, the integrated intensity cut of $\mathrm{CO}$ and isotopologues is presented for different values of $\gamma$ and different beam sizes. The figure shows that isotopologue lines indeed directly trace the gas surface density inside the dust-free 60 AU cavity. In the outer disk, however, shielding effects by dust become important leading to a slightly different $\mathrm{CO}$ abundance and it is difficult to trace the column density to the required accuracy to determine $\gamma$.

\subsection{How well is the $20 \mathrm{AU}$ radius determined?}

How well can the inner radius of the gas disk at 20 AU be determined? To answer this question, we run models with an inner radius of 15, 20, or $25 \mathrm{AU}$. Models with the surface density profile of the representative and with the surface density profile adjusted 
such that the emission at the stellar position is approximately reproduced are calculated. The model-observation comparison of these models is shown in Fig. 12 in the same way as Fig. 10.

We find that models with an inner cavity of 15 or 25 AU instead of $20 \mathrm{AU}$ are unable to reproduce the observed integrated intensity cuts. Models with the gas surface density profile from the representative model fail to reproduce the integrated intensity at the stellar position, while models with a surface density profile adjusted for the emission at the stellar position over or underproduce the emission at offsets of $0.2^{\prime \prime}-0.5^{\prime \prime}$. We conclude that the drop in the surface density at $20 \mathrm{AU}$ is constrained to better than $\sim 5 \mathrm{AU}$.

\subsection{The presence of an inner gas disk}

From the fastest channels with ${ }^{12} \mathrm{CO}$ detected, we concluded that a gas hole with radius $20 \mathrm{AU}$ surrounds the star. How much gas could be hidden inside this hole, located in a small gas disk close to the star, similar to the warm dust disk needed to reproduce the near infrared excess in the SED? A series of models with $\delta_{20} \mathrm{AU}$ varied, but gas completely removed between $1-20 \mathrm{AU}$ or $10-20 \mathrm{AU}$ is run.

We find that for an inner gas disk size of $10 \mathrm{AU}, \delta_{20 \mathrm{AU}}>$ $8 \times 10^{-3}$ overproduces the central peak, while the spectrum is not affected much (Fig. 13). For $\delta_{20} \mathrm{AU} \sim 8 \times 10^{-3}$, the PAHs in the inner disk shield the disk at $20 \mathrm{AU}$ decreasing the integrated intensity slightly (by $\sim 10 \%$ ). For an inner gas disk size of $1 \mathrm{AU}$, only this effect of the shielding can be seen, and even $\delta_{20} \mathrm{AU}=$ 1 would not produce additional emission at the position of the star. The upper limits on the CO mass of an inner disk are $5 \times$ $10^{23} \mathrm{~g}$ for a $10 \mathrm{AU}$ disk with $\delta_{20 \mathrm{AU}}=8 \times 10^{-3}$ and $3 \times 10^{24} \mathrm{~g}$ for a $1 \mathrm{AU}$ disk with $\delta_{20} \mathrm{AU}=8 \times 10^{-3}$. Limits on the $\mathrm{CO}$ mass derived from $4.7 \mu \mathrm{m}$ CO rovibrational lines (Brown et al. 2012, Fig. 10) are highly dependent on the gas temperature and vary between $10^{30} \mathrm{~g}$ for $100 \mathrm{~K}$ and $10^{19} \mathrm{~g}$ for $1000 \mathrm{~K}$. For gas temperatures $>300 \mathrm{~K}$, predicted by our model, better constraints on the $\mathrm{CO}$ mass inside the $20 \mathrm{AU}$ gas hole can be obtained from the rovibrational lines $\left(<10^{21} \mathrm{~g}\right)$.

\subsection{Prediction for the $\mathrm{CO}$ isotopologue lines}

Figure 11 presents the integrated intensity cuts of the $\mathrm{CO}$ isotopologues ${ }^{12} \mathrm{CO},{ }^{13} \mathrm{CO}, \mathrm{C}^{18} \mathrm{O}$, and $\mathrm{C}^{17} \mathrm{O} J=3-2$ and $J=6-5$ transitions convolved to a beam size (FWHM) of $0.25^{\prime \prime}, 0.1^{\prime \prime}$, and $0.05^{\prime \prime}$ for the representative model. The beam sizes have been chosen to represent the current observations, and ALMA in future Cycles. The detection limit of the full ALMA combining all fifty $12 \mathrm{~m}$ antennas for a $5 \sigma$ detection with $1 \mathrm{~h}$ on-source observation is $46 \mathrm{mJy} \mathrm{km} \mathrm{s}^{-1}(J=6-5)$ and $7.7 \mathrm{mJy} \mathrm{km} \mathrm{s}-1$ $(J=3-2)$. For 18-21 antennas (current observations), the detection limit is a factor of 2.5 higher. The limits have been calculated with the ALMA sensitivity calculator ${ }^{3}$, using a channel width of $0.2 \mathrm{~km} \mathrm{~s}^{-1}$, and an intrinsic line width of $1 \mathrm{~km} \mathrm{~s}^{-1}$. For $J=6-5$, we have assumed good weather conditions (average precipitable water vapor levels $P W V<0.47 \mathrm{~mm}$ ) and for $J=3-2$ average weather conditions $(P W V<0.91 \mathrm{~mm})$.

The Figure shows that for beam sizes of $0.25^{\prime \prime},{ }^{13} \mathrm{CO}$ and $\mathrm{C}^{18} \mathrm{O}$ can be easily detected even in this low-mass disk. At the high frequency of the $J=6-5$ transitions, $\mathrm{C}^{17} \mathrm{O}$ is only strong enough for detection when integrated over a larger region as done in Sect. 3.3. For lower frequency $(J=3-2)$, the $\mathrm{C}^{17} \mathrm{O}$

\footnotetext{
3 https://almascience.nrao.edu/proposing/

sensitivity-calculator
}

is predicted to be detectable, although only at a $5 \sigma$ level. For smaller beam sizes than $0.25^{\prime \prime}$, the detection of the isotopologues is challenging, both for $J=3-2$ and $J=6-5$. The steep drop in integrated intensity at the stellar position for smaller beams shows that high angular resolution is indeed crucial to provide better constraints on the amount of gas inside $20 \mathrm{AU}$ (Sect. 4.3.3). In more typical disks with a higher gas mass, the rare isotopologues can be readily detected by ALMA (Bruderer 2013).

\section{Discussion}

\subsection{The gas mass and surface density profile}

The low total gas mass of IRS 48 with $\sim 1.4 \times 10^{-4} M_{\odot}$ compared to the dust mass of $\sim 1.6 \times 10^{-5} M_{\odot}$ yields a gas-to-dust ratio of only $\sim 10$. Since the dust temperature in this disk around an A-type star is too warm for CO freeze-out (Fig. 8) it is not possible that additional gas is missed due to $\mathrm{CO}$ being frozen out. In comparison to HD 142527, another disk around a young Herbig star showing a strongly lopsided continuum emission (Casassus et al. 2013; Fukagawa et al. 2013), the dust mass of IRS 48 is a factor of 2.7 lower, but this disk also shows indications of a gasto-dust ratio lower than the ISM value of 100 (Fukagawa et al. 2013).

What does the low gas-to-dust ratio $^{4}$ observed towards IRS 48 imply? Since van der Marel et al. (2013a) found evidence of grain growth, a gas-to-dust ratio larger than 100 would be expected if some of the dust has grown beyond the size of a few millimeters. These larger grains are not traced by the dust continuum observations. The low gas mass derived in this work thus suggests that some of the gas is removed from the disk.

One possible mechanism for gas removal is a wind, as driven by photoevaporation. Photoevaporation has a short processing time-scale and would quickly remove all gas inside the dust cavity (e.g., Clarke et al. 2001). In IRS 48, however, we find a decrease in the gas surface density at $60 \mathrm{AU}$ of only a factor of $\sim 10$, thus making photoevaporation as the only mechanism forming the 60 AU cavity very unlikely, given the short processing time-scale. The drop at 20 AU may not be constrained by our observations and could be deep. However, at least X-ray driven photoevaporation models (Owen et al. 2011; Rosotti et al. 2013) fail to explain hole sizes of $20 \mathrm{AU}$ for an accretion rate of $4 \times 10^{-9} M_{\odot} \mathrm{yr}^{-1}$ measured towards IRS 48 (Salyk et al. 2013). This accretion rate is also higher than typical EUV photoevaporation rates (Alexander et al. 2006a,b) ruling out EUV driven evaporation. We thus conclude that in IRS 48 photoevaporation is unlikely the reason for the gap formation at 20 and $60 \mathrm{AU}$.

Another mechanism leading to a lower gas-to-dust ratio is, that gas is bound into one or several giant planets or companions in the dust-depleted region inside 60 AU. The dust trap found towards IRS 48, preventing the dust to drift inwards, could filter the gas from the dust (Zhu et al. 2012). Assuming an initial gasto-dust ratio of 100, the amount of gas that has been removed corresponds to $\sim 1.5 M_{\text {Jupiter }}$, which can easily be bound into one companion or giant planet. The companion or giant planet is however not limited to this mass if the initial gas-to-dust mass ratio was 100 , because some of the dust could currently be bound in grains too large to be observed by continuum emission. It is interesting to note that our proposed surface density profile combined with the calculated temperature structure leads to a positive pressure gradient $(\mathrm{d} p / \mathrm{d} r>0)$ at $60 \mathrm{AU}$ as required to trap

\footnotetext{
4 To calculate the gas-to-dust ratio only the observable dust grains with size below a few millimeters are accounted for.
} 
the dust radially. This positive pressure gradient is found independent of the assumed surface density slope $\gamma$.

\subsection{The gas surface density at the dust trap}

The current observational data do not allow us to directly determine the surface density at the position of the dust trap (van der Marel et al. 2013a), since the ${ }^{12} \mathrm{CO}$ emission is optically thick and the $\mathrm{C}^{17} \mathrm{O}$ emission too weak for mapping. The difference of the ${ }^{12} \mathrm{CO}$ integrated intensity at the dust trap compared to the same position in the north by a factor of $\sim 3$ is quite large (Sect. 3.1). One option to explain this would be a gas temperature difference.

The comparison of a model with gas temperature fixed to the dust temperature to that with calculated gas temperature shows that the ${ }^{12} \mathrm{CO} J=6-5$ emission in the outer disk (>60 AU) emerges from a layer where gas and dust temperature are coupled $\left(T_{\text {gas }}=T_{\text {dust }}\right)$. Thus, in order to decrease the ${ }^{12} \mathrm{CO}$ emission at the dust trap by a factor of 3 , the dust temperature needs to be lower by a factor of 3 , which in turn implies a factor of about $3^{4}=81$ less (continuum) radiation should be intercept by the dust, possibly due to shielding by an asymmetric inner disk. The UV pumped CO rovibrational lines observed by Brown et al. (2012) show indeed a strong north/south gradient in intensity, but the VLT-VISIR image by Geers et al. (2007, Fig. 7) indicates that stellar light is received by the south part of the disk. It is thus unclear how a factor of 3 lower dust temperature in the south can be explained. Since the interpretation of the dust trap requires an overdensity of a factor of $2-3$ at the position of the continuum emission, the temperature decrease should actually be even larger. The time-scale to smooth out dust asymmetries is on the order of Myr (Birnstiel et al. 2013), and it could also be that the gas asymmetry that created the dust trap has already disappeared. We conclude that unless the layer where gas and dust temperatures decouple is substantially larger than determined by our models, a temperature difference of a factor of 3 is difficult to explain. Other scenarios for the observed asymmetry based on future observations of optically thin line emission need to be explored. Multiple transition observations in order to determine the gas temperature directly will facilitate this exploration.

\subsection{Consequences of the gas mass for a companion}

Assuming that the $20 \mathrm{AU}$ cavity is carved out by a companion or a giant planet, can we derive constraints on the mass of the companion from the proposed gas surface density profile? For a detailed analysis, hydrodynamical models need to be run in which the depth of the gap in the model is compared to that observed, which is beyond the scope of the study. We only note here that a one Jupiter mass planet is already able to carve out a gap with a depth of a factor of 10 to 1000 , depending on the viscosity parameter (Pinilla et al. 2012). For higher masses, the gravitational torque is more important than the viscous torque and the depth of the gap carved out by a $9 M_{\text {Jupiter }}$ mass is about a factor of 1000, independent of viscosity. Relating the drop in surface density to the planet mass thus requires additional constraints on the viscosity, which are observationally difficult to obtain. In order not to stir up the gas too much and allow dust to be azimuthally trapped, van der Marel et al. (2013a) propose a low viscosity parameter $\alpha_{\mathrm{t}}=10^{-4}$. With such a low $\alpha_{\mathrm{t}}$, already a giant planet with 1 Jupiter mass could carve out the upper limit on the gap depth at $20 \mathrm{AU}$ in IRS 48.
Hydrodynamical models of planets embedded in disks predict a gap opening as far out as $\sim 2$ times the orbital radius of the planet (e.g., Pinilla et al. 2012). A companion situated at $20 \mathrm{AU}$ is thus unlikely to create the observed drop in surface density of a factor $>10$ at 60 AU. Possibly, IRS 48 harbors a multiple system as proposed for other transitional disks (Dodson-Robinson \& Salyk 2011; Zhu et al. 2011) with a more massive planet inside $20 \mathrm{AU}$ and one or several less massive planets between 20 and $60 \mathrm{AU}$. More generally, the drops in the gas density structure such as derived here are potentially the best indicators of just formed planets that are too faint to detect directly.

\section{Conclusions}

In this work, we have analyzed high-resolution ALMA submillimeter observations of ${ }^{12} \mathrm{CO}$ and $\mathrm{C}^{17} \mathrm{O} \mathrm{J}=6-5$ rotational emission from a transition disk using a chemical-physical model. With the model we can set constraints on the surface density profile and the total gas mass of the disk. We can furthermore set constraints on the gas inside a 20 AU cavity and in the 20-60 AU gap, which are thought to be carved out by a companion or one or more giant planets. The main conclusions of our work are:

- The IRS 48 disk has a much lower gas mass $(\sim 1.4 \times$ $10^{-4} M_{\odot}$ ) than previously derived. Together with the dust mass of $\sim 1.6 \times 10^{-5} M_{\odot}$, the gas mass leads to a gas-to-dust ratio of only 10 . Given the uncertainties in determining the gas and dust mass of both a factor of a few, this gas-to-dust ratio is however only marginally below the ISM value of 100 . The low gas-to-dust ratio is possibly related to gas filtered from dust at the position of the dust trap, combined with gas inside the dust trap bound into a companion or giant planet.

- Inside a radius of $60 \mathrm{AU}$, where small dust is thought to be absent, we find that a decrease in the gas surface density profile of a factor of $\sim 12$ is required to reproduce observations. Inside a radius of $20 \mathrm{AU}$, where no $\mathrm{CO}$ rotational emission is detected, a drop of at least an additional factor of $\gtrsim 10$ is derived, in total a drop of a factor of $\gtrsim 110$. To set more stringent constraints, higher resolution observations are required. Observations with beam size on the order of $0.1^{\prime \prime}$ are predicted to be sufficient to constrain the drop considerably better. The drops at $20 \mathrm{AU}$ and $60 \mathrm{AU}$ are derived assuming a slope $\gamma=1$ of the surface density profile $\left(\Sigma \propto r^{-\gamma}\right)$. For $\gamma=0$ and $\gamma=2$, which are also consistent with the data, the drops are a factor of 3 smaller/larger.

- The inner radius of gas depletion at $20 \mathrm{AU}$ can be constrained to better than \pm 5 AU.

- Fitting the SED and the $18.7 \mu \mathrm{m}$ dust continuum image taken by VLT-VISIR requires a small $(<1 \mathrm{AU})$ dusty disk close to the star and a large scale height of the outer disk (scale-height angle $h_{60} \mathrm{AU}=0.14$ radians).

- Despite of the low gas-mass of IRS 48, the full ALMA combining all 50 antennas is predicted to readily detect ${ }^{13} \mathrm{CO}$ $J=6-5$ and $\mathrm{C}^{18} \mathrm{O} J=6-5$. These optically thin isotopologue lines will help to better constrain the gas surface density profile of the disk and to study different scenarios of azimuthal asymmetries.

- The derived gas surface density profile points to the clearing of the cavity by one or several massive planets or companions rather than just photoevaporation or grain-growth.

Acknowledgements. This paper makes use of the following ALMA data: ADS/JAO.ALMA\#2011.0.00635.S. ALMA is a partnership of the European Southern Observatory (ESO) (representing its member states), NSF (USA), 
and National Institutes of Natural Sciences (Japan), together with the National Research Council (Canada) and National Science Council and Academia Sinica Institute of Astronomy and Astrophysics (Taiwan), in cooperation with the Republic of Chile. The Joint ALMA Observatory is operated by the ESO, Associated Universities Inc./National Radio Astronomy Observatory, and National Astronomical Observatory of Japan. We thank the anonymous referee, Til Birnstiel, Joanna Brown, Cornelis Dullemond, Greg Herczeg, Attila Juhàsz, Paola Pinilla, Markus Schmalzl, and Malcolm Walmsley for useful comments and discussions. S.B. acknowledges a stipend by the Max Planck Society and N.v.d.M. is supported by the Netherlands Research School for Astronomy (NOVA) Band 9 funding. T.v.K thanks Allegro, funded by NWO Physical Sciences ("the Netherlands Organization for Scientific Research (NWO), Physical Sciences"), for financial and technical support. We thank Melissa McClure for providing the Spitzer data in electronic form. Astrochemistry in Leiden is supported by the Netherlands Research School for Astronomy (NOVA), by a Royal Netherlands Academy of Arts and Sciences (KNAW) professor prize, and by the European Union A-ERC grant 291141 CHEMPLAN.

\section{References}

Alexander, R. D., \& Armitage, P. J. 2007, MNRAS, 375, 500

Alexander, R. D., Clarke, C. J., \& Pringle, J. E. 2006a, MNRAS, 369, 216

Alexander, R. D., Clarke, C. J., \& Pringle, J. E. 2006b, MNRAS, 369, 229

Andrews, S. M., \& Williams, J. P. 2007, ApJ, 671, 1800

Andrews, S. M., Wilner, D. J., Espaillat, C., et al. 2011, ApJ, 732, 42

Armitage, P. J. 2011, ARA\&A, 49, 195

Birnstiel, T., Dullemond, C. P., \& Pinilla, P. 2013, A\&A, 550, L8

Boogert, A. C. A., Hogerheijde, M. R., Ceccarelli, C., et al. 2002, ApJ, 570, 708

Brown, J. M., Blake, G. A., Dullemond, C. P., et al. 2007, ApJ, 664, L107

Brown, J. M., Blake, G. A., Qi, C., et al. 2009, ApJ, 704, 496

Brown, J. M., Herczeg, G. J., Pontoppidan, K. M., \& van Dishoeck, E. F. 2012, ApJ, 744, 116

Brown, J. M., Pontoppidan, K. M., van Dishoeck, E. F., et al. 2013, ApJ, 770, 94

Bruderer, S. 2013, A\&A, 559, A46

Bruderer, S., van Dishoeck, E. F., Doty, S. D., \& Herczeg, G. J. 2012, A\&A, 541, A91

Calvet, N., D'Alessio, P., Hartmann, L., et al. 2002, ApJ, 568, 1008

Casassus, S., van der Plas, G., Perez, M. S., et al. 2013, Nature, 493, 191

Clarke, C. J., Gendrin, A., \& Sotomayor, M. 2001, MNRAS, 328, 485

Cutri, R. M., Skrutskie, M. F., van Dyk, S., et al. 2003, VizieR Online Data Catalog, II/246

Dodson-Robinson, S. E., \& Salyk, C. 2011, ApJ, 738, 131

Draine, B. T., \& Li, A. 2007, ApJ, 657, 810

Dullemond, C. P., \& Monnier, J. D. 2010, ARA\&A, 48, 205

Dutrey, A., Guilloteau, S., Piétu, V., et al. 2008, A\&A, 490, L15

Dzyurkevich, N., Flock, M., Turner, N. J., Klahr, H., \& Henning, T. 2010, A\&A, 515, A70

Espaillat, C., Furlan, E., D’Alessio, P., et al. 2011, ApJ, 728, 49

Fedele, D., Bruderer, S., van Dishoeck, E. F., et al. 2013, A\&A, 559, A77

Flaherty, K. M., Muzerolle, J., Rieke, G., et al. 2012, ApJ, 748, 71

Fukagawa, M., Tsukagoshi, T., Momose, M., et al. 2013, PASJ, submitted [arXiv: 1309.7400]

Fung, J., Shi, J.-M., \& Chiang, E. 2013, ApJ, submitted [arXiv: 1310.0156]

Garaud, P. 2007, ApJ, 671, 2091

Geers, V. C., Augereau, J., Pontoppidan, K. M., et al. 2006, A\&A, 459, 545
Geers, V. C., Pontoppidan, K. M., van Dishoeck, E. F., et al. 2007, A\&A, 469, L35

Goto, M., Usuda, T., Dullemond, C. P., et al. 2006, ApJ, 652, 758

Helou, G., \& Walker, D. W. 1988, Infrared astronomical satellite (IRAS) catalogs and atlases vol. 7, 1

Huélamo, N., Lacour, S., Tuthill, P., et al. 2011, A\&A, 528, L7

Isella, A., Pérez, L. M., \& Carpenter, J. M. 2012, ApJ, 747, 136

Isella, A., Pérez, L. M., Carpenter, J. M., et al. 2013, ApJ, 775, 30

Jonkheid, B., Dullemond, C. P., Hogerheijde, M. R., \& van Dishoeck, E. F. 2007, A\&A, 463, 203

Kraus, A. L., \& Ireland, M. J. 2012, ApJ, 745, 5

Kretke, K. A., \& Lin, D. N. C. 2007, ApJ, 664, L55

Loinard, L., Torres, R. M., Mioduszewski, A. J., \& Rodríguez, L. F. 2008, ApJ, 675, L29

Loren, R. B. 1989, ApJ, 338, 902

Lyo, A.-R., Ohashi, N., Qi, C., Wilner, D. J., \& Su, Y.-N. 2011, AJ, 142, 151

Lyra, W., \& Lin, M.-K. 2013, ApJ, 775, 17

Maaskant, K. M., Honda, M., Waters, L. B. F. M., et al. 2013, A\&A, 555, A64

McClure, M. K., Furlan, E., Manoj, P., et al. 2010, ApJS, 188, 75

Mulders, G. D., Paardekooper, S.-J., Panić, O., et al. 2013, A\&A, 557, A68

Murillo, N. M., Lai, S.-P., Bruderer, S., Harsono, D., \& van Dishoeck, E. F. 2013, A\&A, 560, A103

Owen, J. E., Ercolano, B., \& Clarke, C. J. 2011, MNRAS, 412, 13

Pickles, A. J. 1998, PASP, 110, 863

Piétu, V., Dutrey, A., Guilloteau, S., Chapillon, E., \& Pety, J. 2006, A\&A, 460, L43

Pinilla, P., Benisty, M., \& Birnstiel, T. 2012, A\&A, 545, A81

Pontoppidan, K. M., Boogert, A. C. A., Fraser, H. J., et al. 2008, ApJ, 678, 1005

Quanz, S. P., Amara, A., Meyer, M. R., et al. 2013, ApJ, 766, L1

Rice, W. K. M., Armitage, P. J., Wood, K., \& Lodato, G. 2006, MNRAS, 373, 1619

Rosotti, G. P., Ercolano, B., Owen, J. E., \& Armitage, P. J. 2013, MNRAS, 430, 1392

Salyk, C., Blake, G. A., Boogert, A. C. A., \& Brown, J. M. 2009, ApJ, 699, 330

Salyk, C., Herczeg, G. J., Brown, J. M., et al. 2013, ApJ, 769, 21

Strom, K. M., Strom, S. E., Edwards, S., Cabrit, S., \& Skrutskie, M. F. 1989, AJ, 97, 1451

Thi, W. F., Ménard, F., Meeus, G., et al. 2013, A\&A, 557, A111

van der Marel, N., van Dishoeck, E. F., Bruderer, S., et al. 2013a, Science, 340, 1199

van der Marel, N., van Dishoeck, E. F., Bruderer, S., \& van Kempen, T. A. 2013b, A\&A, submitted

van der Plas, G., van den Ancker, M. E., Acke, B., et al. 2009, A\&A, 500, 1137

van Kempen, T. A., van Dishoeck, E. F., Salter, D. M., et al. 2009, A\&A, 498, 167

Weingartner, J. C., \& Draine, B. T. 2001, ApJ, 548, 296

Whipple, F. L. 1972, in From Plasma to Planet, ed. A. Elvius (New york: Wiley Interscience Division), 211

Wright, E. L., Eisenhardt, P. R. M., Mainzer, A. K., et al. 2010, AJ, 140, 1868

Yamamura, I., Makiuti, S., Ikeda, N., et al. 2010, VizieR Online Data Catalog: II/298

Zacharias, N., Monet, D. G., Levine, S. E., et al. 2005, VizieR Online Data Catalog: II/297

Zhu, Z., Nelson, R. P., Hartmann, L., Espaillat, C., \& Calvet, N. 2011, ApJ, 729, 47

Zhu, Z., Nelson, R. P., Dong, R., Espaillat, C., \& Hartmann, L. 2012, ApJ, 755, 6 
S. Bruderer et al.: Gas structure inside dust cavities of transition disks
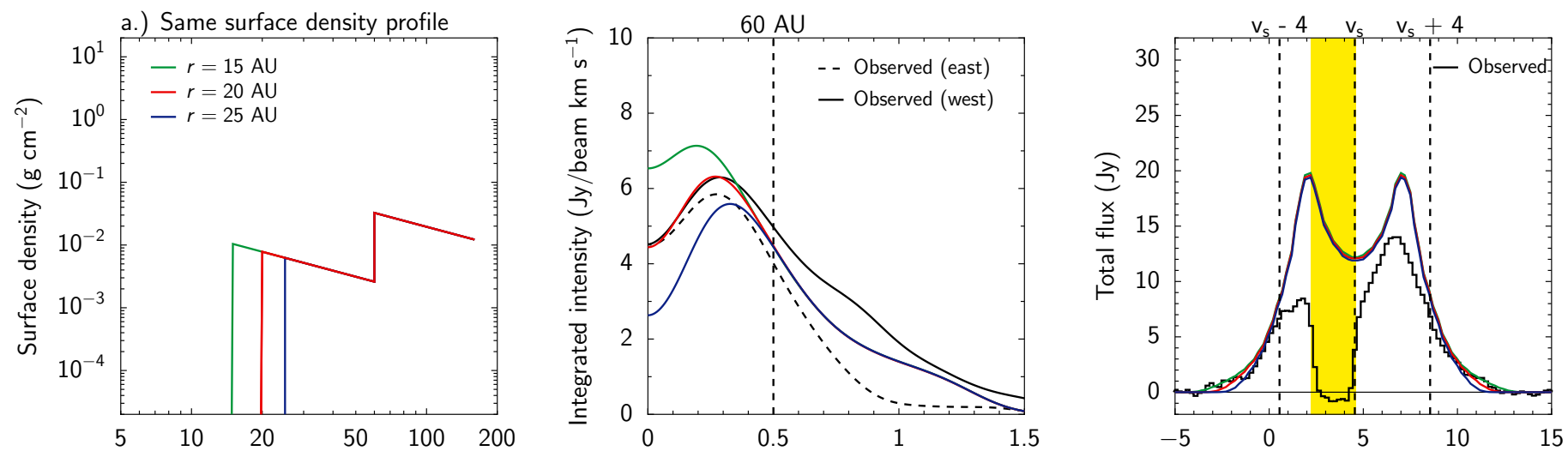

b.) Changed surface density profile
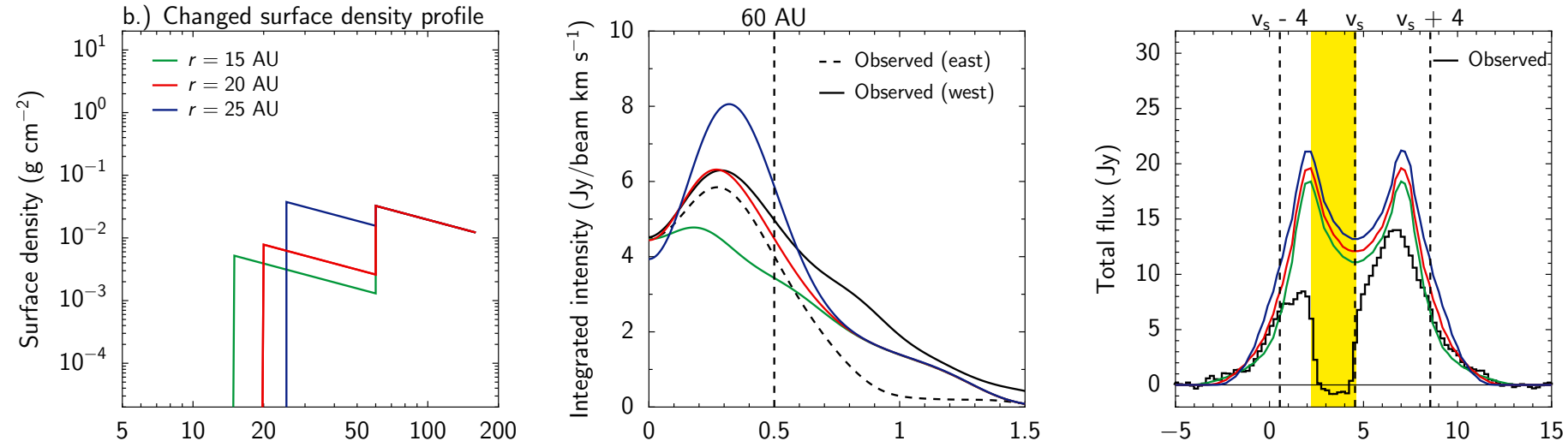

Fig. 12. Comparison of modeled and observed ${ }^{12} \mathrm{CO} J=6-5$ intensity profiles and spectra, showing the effect of changing the inner radius of the gas disk at around $20 \mathrm{AU}$. Left panels: adopted surface density profiles with variations of the representative model. The red line is the representative model. Center panels: intensity profile extracted along the major axis of the disk. The vertical dashed line indicates the radius of the dust-free cavity. Right panels: total spectrum. The yellow region indicates regions affected by the foreground. Vertical dashed lines show the source velocity $v_{\mathrm{s}}=4.55 \mathrm{~km} \mathrm{~s}^{-1}$ and velocities $v_{\mathrm{s}} \pm 4 \mathrm{~km} \mathrm{~s}^{-1}$ representing velocity ranges corresponding to the inner disk (radii $\lesssim 60$ AU). a) (Upper row) using the gas surface density profile of the representative model. b) (Lower row) by varying the surface density such that the emission at the stellar position is approximately reproduced. 

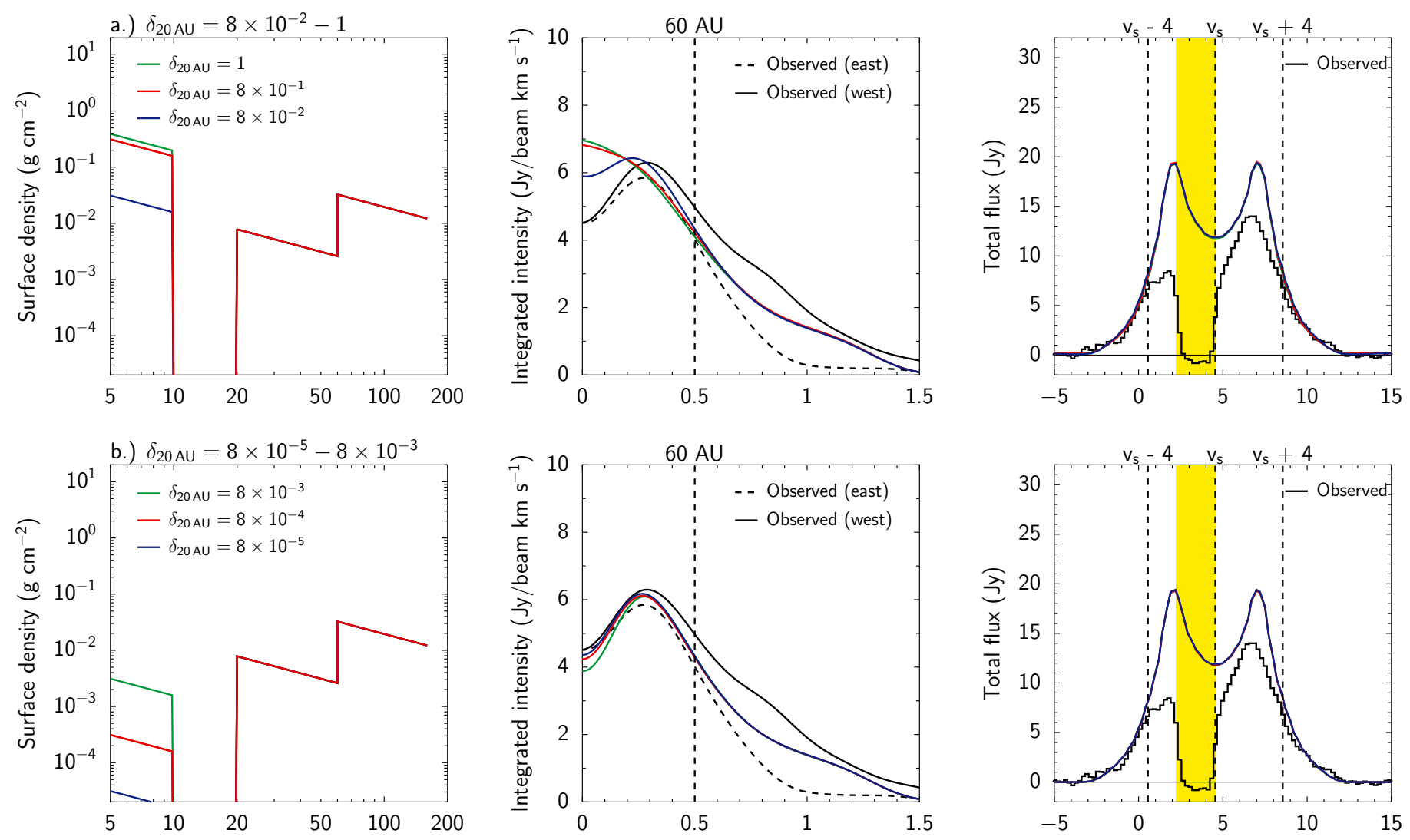

Fig. 13. Comparison of modeled and observed ${ }^{12} \mathrm{CO} J=6-5$ intensity profiles and spectra, showing the effect of an inner gas disk extending from 0.4-10 AU. The surface density profile outside $20 \mathrm{AU}$ is set to the representative model. Left panels: adopted surface density profiles with variations of the representative model. The red line is the representative model. Center panels: intensity profile extracted along the major axis of the disk. The vertical dashed line indicates the radius of the dust-free cavity. Right panels: total spectrum. The yellow region indicates regions affected by the foreground. Vertical dashed lines show the source velocity $v_{\mathrm{s}}=4.55 \mathrm{~km} \mathrm{~s}^{-1}$ and velocities $v_{\mathrm{s}} \pm 4 \mathrm{~km} \mathrm{~s}^{-1}$ representing velocity ranges corresponding to the inner disk (radii $\lesssim 60 \mathrm{AU})$. a) (Upper row) adopting $\delta_{20} \mathrm{AU}=8 \times 10^{-2}-1$. b) (Lower row) adopting $\delta_{20} \mathrm{AU}=8 \times 10^{-5}-8 \times 10^{-3}$.
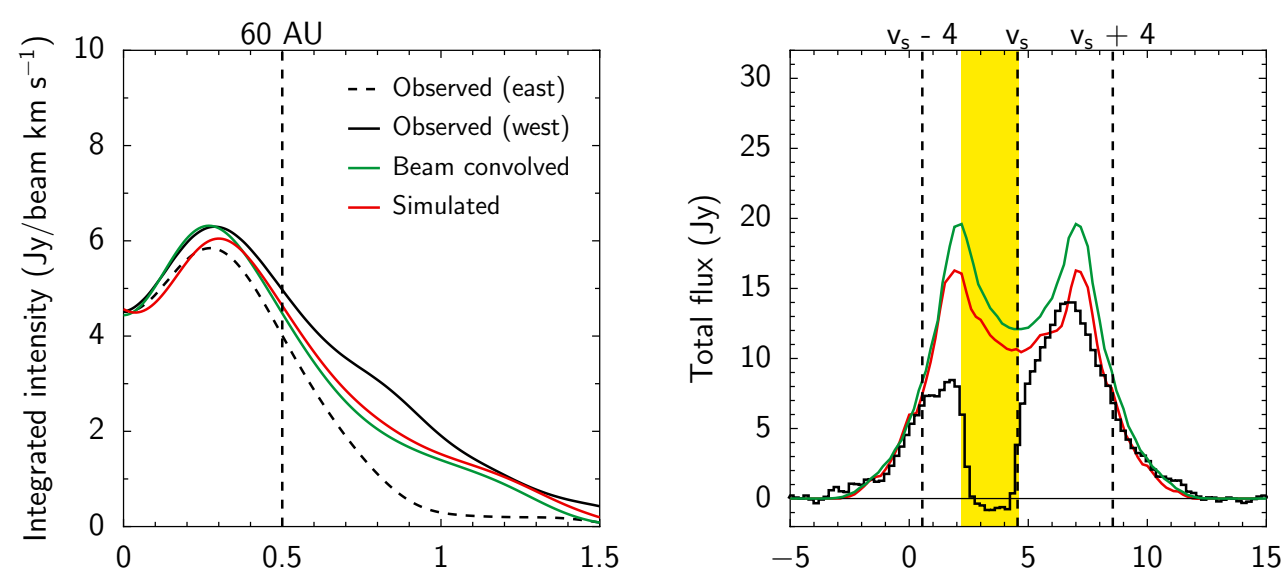

Fig. 14. Comparison of the intensity profile and total spectrum of the representative model convolved to the ALMA beam or simulated using the observed $(u, v)$-coverage. Left panel: intensity profile extracted along the major axis of the disk. The vertical dashed line indicates the radius of the dust-free cavity. Right panel: total spectrum. The yellow region indicates regions affected by the foreground. Vertical dashed lines show the source velocity $v_{\mathrm{s}}=4.55 \mathrm{~km} \mathrm{~s}^{-1}$ and velocities $v_{\mathrm{s}} \pm 4 \mathrm{~km} \mathrm{~s}^{-1}$ representing velocity ranges corresponding to the inner disk (radii $\lesssim 60$ AU). 
S. Bruderer et al.: Gas structure inside dust cavities of transition disks
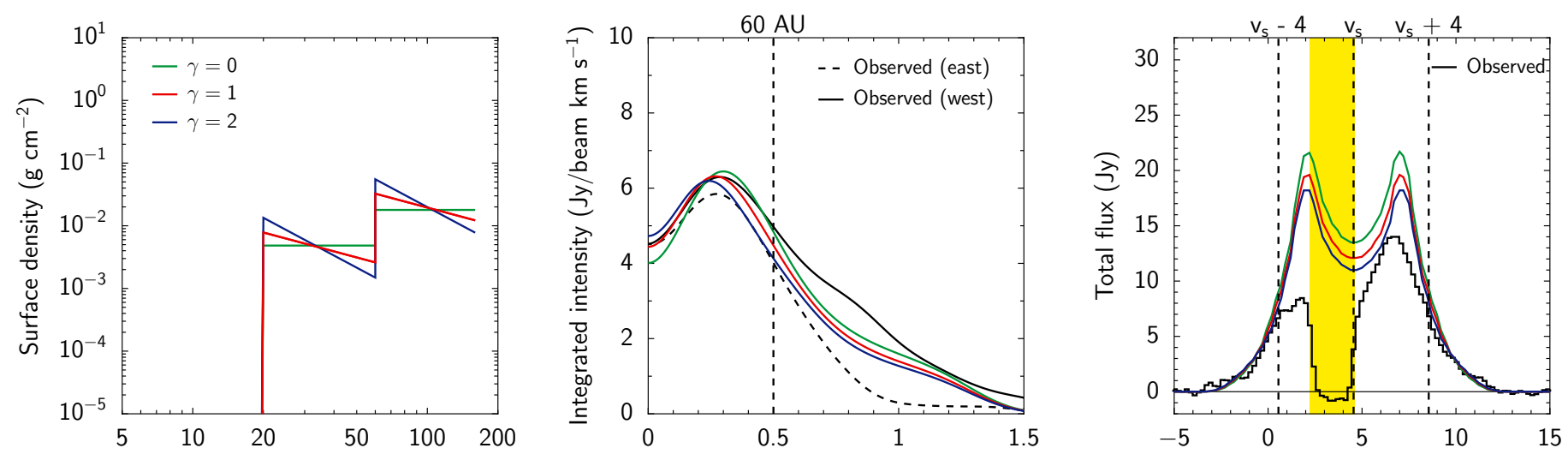

Fig. 15. Comparison of modeled and observed ${ }^{12} \mathrm{CO} J=6-5$ intensity profiles and spectra, showing the intensity profile and total spectrum of models with different slope $\gamma$ of the gas and dust surface density profile $\left(\Sigma \propto r^{-\gamma}\right)$. Left panel: adopted surface density profiles with variations of the representative model. The red line is the representative model. Center panel: intensity profile extracted along the major axis of the disk. The vertical dashed line indicates the radius of the dust-free cavity. Right panel: total spectrum. The yellow region indicates regions affected by the foreground. Vertical dashed lines show the source velocity $v_{\mathrm{s}}=4.55 \mathrm{~km} \mathrm{~s}^{-1}$ and velocities $v_{\mathrm{s}} \pm 4 \mathrm{~km} \mathrm{~s}^{-1}$ representing velocity ranges corresponding to the inner disk (radii $\lesssim 60 \mathrm{AU}$ ). 